\title{
AIAA 96-1987
}

Four-Dimensional Measurements of Vector Fields in Turbulent Flows (Invited Paper)

Werner J.A. Dahm, Lester K. Su and Kathleen M. Tacina

Laboratory for Turbulence and Combustion (LTC)

Department of Aerospace Engineering

The University of Michigan

Ann Arbor, MI

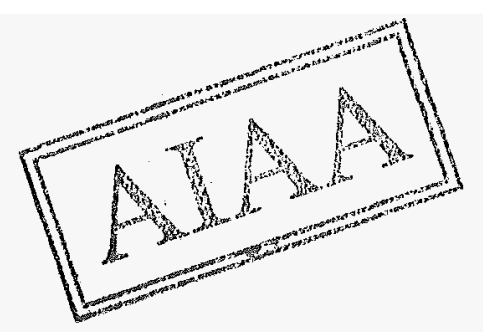

\section{7th AIAA Fluid Dynamics Conference June 17-20, 1996 / New Orleans, LA}




\title{
Four-Dimensional Measurements of Vector Fields in Turbulent Flows
}

\author{
Werner J.A. Dahm1, Lester K. Su² and Kathleen M. Tacina ${ }^{3}$ \\ Laboratory for Turbulence and Combustion (LTC) \\ Department of Aerospace Engineering \\ The University of Michigan \\ Ann Arbor, MI 48109-2118
}

\begin{abstract}
Experimental methods and results are presented for fully-resolved, three- and four-dimensional,spatio-temporal measurements of scalar gradient vector fields $\nabla \zeta(\mathrm{x}, t)$ and velocity vector fields $\mathrm{u}(\mathrm{x}, t)$ in turbulent flows. Each three-dimensional spatial data volume is composed of up to $256^{3}$ spatial data points, with volumes acquired sequentially in time. The four-dimensional data sets are each comprised of over 3 billion individual point measurements, and are simultaneously differentiable in $\mathrm{x}, \mathrm{y}, \mathrm{z}$, and $t$, allowing access to the spatial structure and temporal dynamics in these fields. Space and time scales relevant to such measurements are summarized. A method for assessing the resolution achieved by such measurements is presented, and as are criteria for over-resolution in digital measurements. Results give the space- and time-varying conserved scalar field and vector velocity field simultaneously on a regular three-dimensional spatial grid. Direct differentiation of these fields yields the spatial structure in the full nine-component velocity gradient tensor field $\nabla \mathbf{u}(\mathbf{x}, l)$. From these, the vector vorticity field $\omega_{i}(\mathbf{x}, t)$ and tensor strain rate field $\varepsilon_{i j}(\mathbf{x}, t)$ are extracted, as are the kinetic energy dissipation rate field $2 \vee \varepsilon: \varepsilon(\mathbf{x}, t)$, the enstrophy field $1 / 2 \omega \cdot \omega(\mathrm{x}, t)$, the enstrohy production rate field $\omega \cdot \varepsilon \cdot \omega(\mathrm{x}, t)$ and the pressure gradient field $\nabla p(x, l)$. Extension of the scalar imaging velocinietry technique to whole-field measurements are described, and various limiting cases is described, which yields velocity vector fields that are filtered in space and time at the resolution scale A. Such whole-field SIV measurementsallow use of the full spatial and temporal dynamic range available to the measurements, and permits measurements in turbulent flows at arbitrarily high Reynolds numbers.
\end{abstract}

"If we are to achieve results never before accomplished, we must expect no employ methods never before attempted."

Sir Francis Bacon

\section{INTRODUCTION}

Insights into the fully-resolved, three-dimensional, spatial structure and simultaneous temporal dynamics of the full ninecomponent velocity gradient tensor field $\nabla \mathbf{u}(\mathbf{x}, t)$ at the small scales of turbulent flows are key to developing an understanding of the physics of turbulence and to the development of models for these small scales in large eddy simulations. These small scales are generally presumed to be quasi-universal in high Reynolds number flows, and are thus studied in a generic context. However, laboratory experiments under controlled conditions capable of directly yielding useful information on the detailed structure and dynamics of these scales have been few, and as a consequence such studies have been generally limited to direct numerical simulations under idealized conditions.

To date most experiments of this type have been limited to single-point measurements of a small subset of the full velocity gradient tensor field. The earliest and still most widely used technique for measuring one or several of the gradient tensor components relies on multiple hot-wire or hot-film probes. Such probes have been in use since theoriginal four-wire probe was developed by Kovasznay (1954) for measurements of the

I Associate Professor; AIA A Member; Corresponding author.

2 Present address: University of Texas at Austin; AIAA Member

3 AlAA Student Member.

Copyright (1) 1996 by Werner J.A. Duhm streaniwise component of the vorticity vector at a single spatial point. Surveys of more modern probes and data obtained from them are given by Wallace (1986) and Foss \& Wallace (1989). Since then, Vukoslavcevic, Wallace \& Balint (199I) and Balint, Wallace\& Vukoslavcevic (199I) have presented results from a nine-sensor hot-wire probe that yields simultaneous measurementsof all threevorticity components. Kit et al (1987. 1988) also developed a nine-wire probe to measure several components of the velocity gradient tensor, and Tsinober, Kit \& Dracos (1992) assembled a 12-sensor hot-wire probe, as well as a 20sensor probe without common prongs, to measure all nine components of the velocity gradient tensor at a single spatial point. Such multiple hot-wire/film probes have provided otherwise inaccessible insights into the structure and dynamics in the velocity gradient tensor field in turbulent flows, especially as regards statistics of various quantities of interest. However, as the number of sensor wires and prongs in these probes increases, blockage effects can become significant and alter velocity gradients in the vicinityof the probe tip. Moreover, such probes inherently allow measurements at only a single spatial point. Spatial structure in the velocity gradient component fields is accessible only if a Taylor hypothesis is accepted, and even then is only available along one spatial dimension.

For these reasons, non-invasive optical techniques have been under development for several years to measure components of the velocity gradient tensor field in turbulent flows. These techniques are making incrcasing use of advanced laser diagnostics, 
high-speed imaging arrays. and high-speed data acquisition capabilities to facilitate a variety of optically-based measurement techniques that provide information over spatial fields of many points. Reviews of some of these are given. for example, by Adrian (1986. I99 I. 1996), Lauterborn \& Vogel (1984), and Miles \& Nosenchuck (1989). Such techniques potentially offer high spatial and temporal resolution, as well as genuine spatial field information in place of classical single-point time-series data. The most widely used of such methods are panicle tracking and particle image velocimetry (PTV and PIV) techniques. These generally produce two-component velocity vectors over two-dimensional fields, though three-dimensional panicle tracking (e.g. Nishino et al 1989; Kasagi \& Matsunaga 1995) and holographic panicle image velocimetry (e.g. Scherer \& Bemal 1993: Meng \& Hussain 1995) are being developed to measure full, three-component velocity vector fields in complex flows (see also Adrian 1996).

There are. however. two principle difficulties currently facing such particle-based measurement techniques. First. holographic extension of PIV to three-dimensional spatial measurements can be problematic. owing in part to resolution and depthof-field limitations. Second, the high particle seeding densities required to adequately resolve the finest length scales in turbulent tlows can make optical penetration into the flow difficult. Both of these problems can be circumvented by using an effectively conimuous distribution of laser tluorescent dye molecules as the seed. Their size eliminates the Mie scattering associated

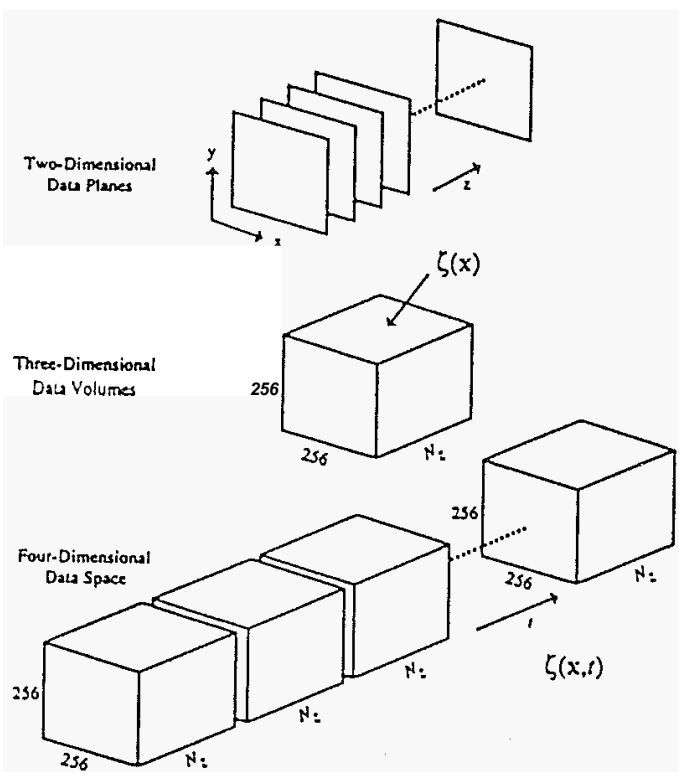

Figure I. Schematic showing the structure of the three- arid fourdimensional data volumes. Each lour-dimensional measurement produces the conserved scalar lield $\zeta(x, t)$ at up to 3.1 billion points in space and time. antanged os a temporal sequenceof three-dimensionalspatial datal volunles. The spatial separation belween adjacent points in $x, y$, and $z$ is smaller than the local strain-linited molecular diffusion scale $\lambda_{0}$ and the temporal separation between the sune spatial point in successive datal volunes is less than the ditfusion scalle advection time $T_{0}$. allowing dilferentiition simulanteously in space and time. The vector velocity field $u(x, n)$ is obtained by inverting the scalar transport equation for the given scalar tield dati. together with the smoothness constraint between $\lambda_{0}$ and $\lambda_{v}$ with discrere panicles. thus niaintaininp optical transparency in the How field. Moreover. currently-available scanning techniques can be readily used to obtain three-dimensional spatial information and simultaneous temporal information. as will be described below. In such scalar-based velocimetry techniques. the dye molecules are both dynamically passive and conserved. so the conserved scalar transport equation governs their continuum concentration field. Unlike PIV. determining velocities then no longer involves tinding discrete particle displacements. and is instead based on inversion of the space- and time-evolving dye concentration field to extract the underlying velocity field, as will also be described below. Such scalar imaging velocimrtry methods currently allow fully-resolved four-dimensional spatiotemporal measurements of the fine scales of turbulent tlows (Dahm, Su \& Southerland 1991. 1992: Su \& Dahm 1996a,b). Moreover, extensions of this technique are currently underway to permit whole-field scalar imaging velocimetry measurements of turbulent tlows. as will be described below.

Such fully-resolved, three- and four-dimensional, spatiotemporal measurements of the conserved scalar field $\zeta(x, t)$ and velocity field $\mathbf{u}(\mathbf{x}, l)$ in turbulent llows are based on highresolution scanning planar laser induced tluorescence imaging from the concentration tield of a passive water-solubledye (disodium tiuorescein) having $S c=2075$, combined with continuous high-speed acquisition of gigabyte-sized data sets. Details of the measurement technique are given by Dahin, Southcrland \& Buch (1991). Buch \& Dahin (1996). and Southerland \& Dahm (1994. 1996). The resolution achieved reaches below the local strain-limited molecular diffusion scale in all three spatial dimensions as well as in time. The resulting threc-dimensional data sets are comprised of nearly 200 temporally successive spatial data volumes, each of which consists of up to $256^{3}$ spalial data points arranged on a regular grid as shown in Figure I. Owing to the high resolution and signal quality attained, the restilting data are fully difterentiable in $x . y$, and $z$. The fourdimensional data sets are each comprised of over 3 billion individual point measurements throughout a smaller spatialdata volume, and are simultaneously differentiable in $\mathbf{x}, y, z$. and $I$. as also shown in Figure I, allowing access to the spatial structure and temporal dynamics of the full scalar gradient vector lield $\nabla \zeta(x, t)$ at the small scales of a turbulent llow. Key components of the measurement system are shown in Figure 2.

Velocimetry techniques based on such scalar field measurements were first introduced by Dahm. Su \& Southerland (1991, 1992). In the scalar imaging velocimetry (SIV) method, the velocity field $u(x, t)$ is deterinined by invering the exact scalar transpon equation. namely

$$
\mathbf{u} \cdot \nabla \zeta=-\left[\frac{\partial}{\partial t}-\frac{1}{R_{\iota} S c} \nabla^{*}\right] \zeta(x, l)
$$

from four-dimensional ineasurernents ot' the scalar field $\zeta(x, t)$ together with one or more additional physical constraints such as the smoothness (length scalc) relation between the velocity and scalargradiont vector fields in turbulent flows. As was pointed out by Dahm, Su \& Southcrland (1992). the latter point recog. nizes that. in addition to the true velocity ficld $u(x, l)$. (I) admits all other velocity fields with streamlinesconlined to isoscalar 


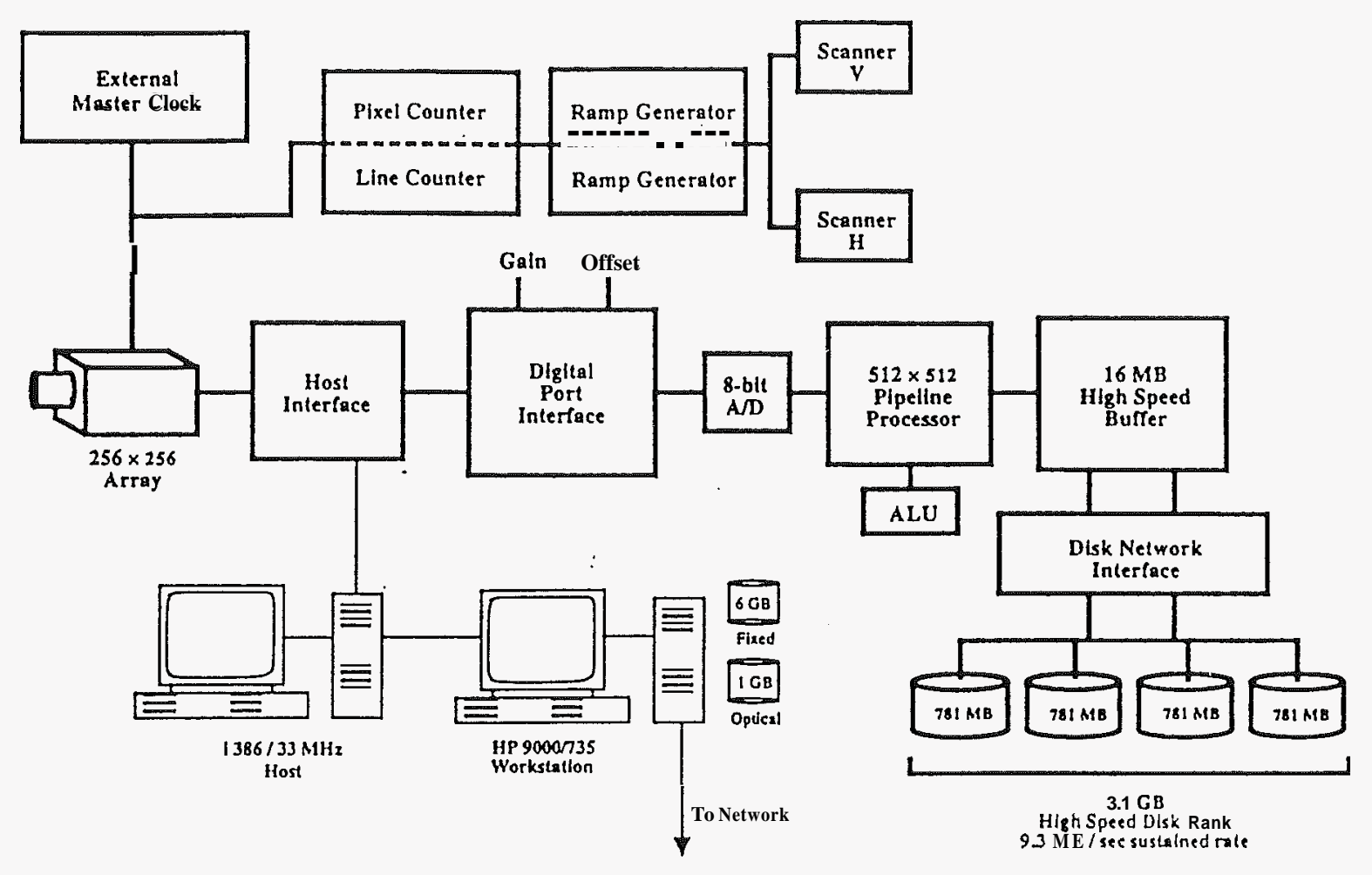

Figure 2. Key components of the measurement system assembled for the present fully-resolved. four-dimensional. spatio-temporal. laboratory meilsurements of the conserved scalar field $\zeta(x, t)$ and the velocity tield $\mathbf{u}(\mathbf{x} . t)$ at the sinall scales of turbulent shear tlows. Two low-intertia galvanometric mirror scamters are slaved to the imaging array timing to rapidlysweepa laser beam in a raster fashion through a stmall three-dimensional spitial volume in the llow. The data acquisition system can achieve sustained data throughput rates up to $9.3 \mathrm{MB} / \mathrm{sec}$ for volumes as large as the full 3.1 GB disk capacity.

surfaces. since then $\mathbf{u} \cdot \nabla \zeta \equiv 0$. However in turbulent flows these spurious velocity fields can be excluded. since the ratio of smallest length scales in the true velocity field and the scalar field differ by a factor $S c^{1 / 2}$, while all the spurious velocity fields would contain length scales as small as those in the scalar field. The smoothness constraint' thus permits the full velocity vector field to be extracted despite the fact that only the component of $u(x, t)$ along the local $\nabla \zeta(x, t)$ direction is involved in the scalar field dynamics. This can be done via direct inversion of (1) or by a variational implementation, as described by $\mathrm{Su} \& \mathrm{Dahm}$ $(1996 a, b)$.

Since the concept of scalar-based velocimetry was first presented. a number of related techniques have been suggested for obtaining velocity fields from scalar field data. A iwo-scalar approach was suggested by Pearlstein \& Carpenter (1995) that would not require the smoothness constraint inherent in SIV. However that method requires developmentofa means to simultaneously measure two scalar fields. and requires that the two scalar gradient vectors not align. A single-scalar approach based

It is this point that was missed in the criticism by Pearlstein \& Carpenter (199.5), whose proot merely showed that in the absence of such additional conseraints the scalar transport equation alone is not sufficient to uniquely determine $u(x, I)$. This fact is readily apparent from (I), and was noted by Dahm, Su \& Southerland (1992). on optimal litting of velocities and velocity gradients to niatch the scalar field evolution has been proposed by Maas (1993) and applied in turbulentjets by Merkel 1995. Merkel et al 1995, and Dracos et al $1995^{2}$. The method does not make use of the scalar transport equation, and instead finds the velocity field that maximizes the correlation between the measured scalar tield at successive times. Note that the admissibility of spurious velocity fields. with streamlines on isoscalar surfaces. is inherent in all velocimetry methods based on a single scalar. In the correlation method. a smoothness constraint somewhat analogous to that in the present SIV method is indirectly introduced through the template on which the correlations are computed.

The present paper summarizes the scalar field measurement techniqueand the inversion technique used to obtain the full vector velocity fields, and presents sample data from such measurements. These include the conserved scalar field $\zeta(x, 1)$, the scalargradient vector field $\nabla \zeta(x, 1)$, the velocity field $u(x, 1)$, the vector vorticity field $\omega_{i}(x, l)$ and tensor strain rate field $\varepsilon_{i j}(x, l)$. the kineticenergy density field $k(x, 1)$, the kinetic energy dissipa-

2 Muas ( 1993) refers to the approach as Adaptive Least Squares Correlation. The sime technique wits proposed later, and apparently independently. by Tokumaru \& Dimotakis (1995), who reler to it as Image Correlation Velocimetry. Neither addresses the uniqueness issue explicitly or introduces additional physical constraints to exclude the spurious velocity fields. 
tion rate field $\Phi(\mathbf{x}, t)$, the enstrophy field $W(\mathbf{x}, t)$, and the pressure gradient field $\nabla p(\mathrm{x}, t)^{\prime}$. The present paper also discusses the extension of this scalar imaging velocimetry technique to whole-field measurements of such quantities in turbulent flows.

\section{SPACE AND TIME SCALES}

Results of the type to be presented below rely crucially on the resolution achieved relative to certain critical space and time scales in scalar gradient vector fields $\nabla \zeta(x, t)$ and velocity gradient tensor fields $\nabla \mathbf{u}(\mathbf{x}, t)$ in turbulent flows. Since turbulent shear tlows are characterized by a wide range of length and time scales over which variations occur, there are many scales used in the literature to demark various subranges over which distinctly different physical processes dominate. However, only a few of these are of key relevance to measurements of the present type, as summarized below.

\section{Ottrer Scules}

In shear-driven turbulent flows, the local outer length and velocity scales $u$ and 6 are those that characterize the local mean shear profile. For example, in jets and plumes these are the local mean centerline velocity and the local flow width, while in shear layers the relevant quantities are the freestream velocity difference and the local flow width. All quantities associated with the outer scales are properly normalized by $u$ and 6 , thus for example the local outer time scale is $\tau_{\delta} \equiv \delta / u$. The local outer-scale Reynolds number $R e_{\delta} \equiv u \delta / \mathrm{V}$ then properly scales the local turbulence properties of the flow. Key among these for the present purposes is the relation between the local outer scales and the local inner scales.

Working in local outer scales has several advantages over the more widespread use of flow-specificsource variables, such as the nozzle diameter and exit velocity in the case of jets. or the density difference at the source in the case of plumes. Such source variables often have at most an indirect influenceon the outer scales. as can be seen from the proper momentum-based scaling laws, and thus at most have an indirect and potentially confusing intluence on the local turbulence properties. Indeed, there are many examples in the literature where use of source variables has led to erroneous conclusions. Moreover, sufficiently small scales of all turbulent shear tlows at the same local outcr-scale Reynolds number $R e_{\delta}$ have essentially similar structural and statistical properties. Parametrizations and normalizations based on flow-specific variables potentially obscure this quasi-universality and thereby obfuscate one of the strongest organizing principles available in turbulence studies.

\section{Inner Scules}

The inner scales in turbulent flows characterize the finest length scale and finest (Lagrangian) time scale on which variations occur in the flow. The finest length scale results from the competing effects of strain acting to reduce the gradient length scale,

\footnotetext{
3 Some of these results will also be appearing in an upcoming scries of papers on scalur imaging velocimetry (Su \& Dahm 1996a,b).
}

and molecular diffusion acting to increase the gradient scale. These can be shown to reach an equilibriumat the strain-limited viscous diffusion length scale $\lambda_{v}$ in the velocity gradient field. and at the strain-limited scalar diffusion length scale $\lambda_{D}$ in the scalar gradient field. These inner length scales are related to the local outer scale 6 as $\lambda_{v} \approx \mathbf{A} 6 \cdot R e_{\delta}^{-3 / 4}$ and $\lambda_{\mathrm{D}}=\lambda_{v} S C^{-1 / 2}$. The constant $\mathbf{A} \approx I 1.2$ conies from direct measurements of $\lambda_{\mathrm{D}}$ by Buch \& Dahm $(1996 a, b)$ and Southerland \& Dahm (1996). where $\delta$ is the full width over which the mean velocity profile drops to $5 \%$ of its peak value, and $\lambda_{\mathrm{D}}$ is the average distance over which the layer-normal scalar dissipation profiles drop to $20 \%$ of theirpeak value. As noted above. when working in the local outer-scale Reynolds number $R e_{\delta}$ the value of $\Lambda$ should be universal; if working in source-based Reynolds numbers it will appear to depend on the flow.

Note that the viscous diffusion scale $\lambda_{v}$ is directly proportional to the classical Kolniogorov length scale $\lambda_{K} \equiv\left(v^{3} / \varepsilon\right)^{1 / 4} \mathrm{de}-$ fined in terms of the mean dissipation rate $\varepsilon$. Using the dissipation results in turbulentjets of Friehe, van Atta \& Gibson (197I) and $\mathbf{A}$ as above gives $\lambda_{\mathrm{v}} \approx 5.9 \lambda_{\mathrm{K}}$. Note that although $\lambda_{\mathrm{K}}$ gives the correct parametric scaling for the finest velocity gradient length scale, it is defined entirely on dimensional grounds and thus does not correspond directly to the resolution requirement.

Similarly, the scalar diffusion length scale $\lambda_{D}$ is simply propor ${ }^{-}$ tional ${ }^{\text {to }}$ the Batchelor scale, but gives the physical thickness of the scalar dissipation layers in a turbulent flow.

Apart from the inner length scale, the viscosity is the only directly relevant physical parameter at the inner scales. and thus the corresponding inner time scale is $\tau_{v}=\left(\lambda_{v}{ }^{2} / v\right)$. This gives the shortest time scale on which the underlying vorticity tield evolves in a Lagrangian frame. The local outer-scale Reynolds number $R e_{\delta}$ then provides the relation to the local outer time scale as $\tau_{v} \approx \Lambda^{2} \tau_{\delta} \cdot R e_{\delta}^{-1 / 2}$, where $\tau_{\delta} \equiv(\delta / u)$. The inner time scale is directly proportional ${ }^{\text {to }}$ the classical Kolmogorov time scale $\tau_{K} \equiv(v / \varepsilon)^{1 / 2}$, where as above $\tau_{K} \approx 35 \tau_{v}$

When the outer scale Reynolds number $R e_{\delta}$ is sufficiently large, the velocity field $\mathbf{u}(\mathbf{x}, t)$ and scalar field $\zeta(\mathbf{x}, t)$ should be independent of $R e_{\delta}$ when viewed on the inner scales. Moreover, since the outer variables enter the governing equations only through $R e_{\delta}$, the velocity and scalar fields should therefore also be independent of the outer scale variables and. as a further consequence, should be independent of the particular shear flow as well. It is in this sense that the fine scale structure of the velocity and scalar fields, when viewed on the inner scales of high Reynolds number turbulent flows. are believed to be largely universal (i.e. independent of the Reynolds number and of the particular tlow).

\section{Advection Scales}

The inner Lagrangian time scale $\tau_{v}$ is not, however, the temporal resolution requirement for turbulent flow measurements. Tlic Eulerian nature of mcasurenicnts obtained at any fixed spatial point introduces the much shorter viscous advection time scale $T_{v} \equiv\left(\lambda_{v} / u\right)$ in the velocity gradient field, and the corresponding scalar advection time scale $T_{\mathrm{D}} \equiv\left(\lambda_{\mathrm{D}} / u\right)$ in the scalar gradient 

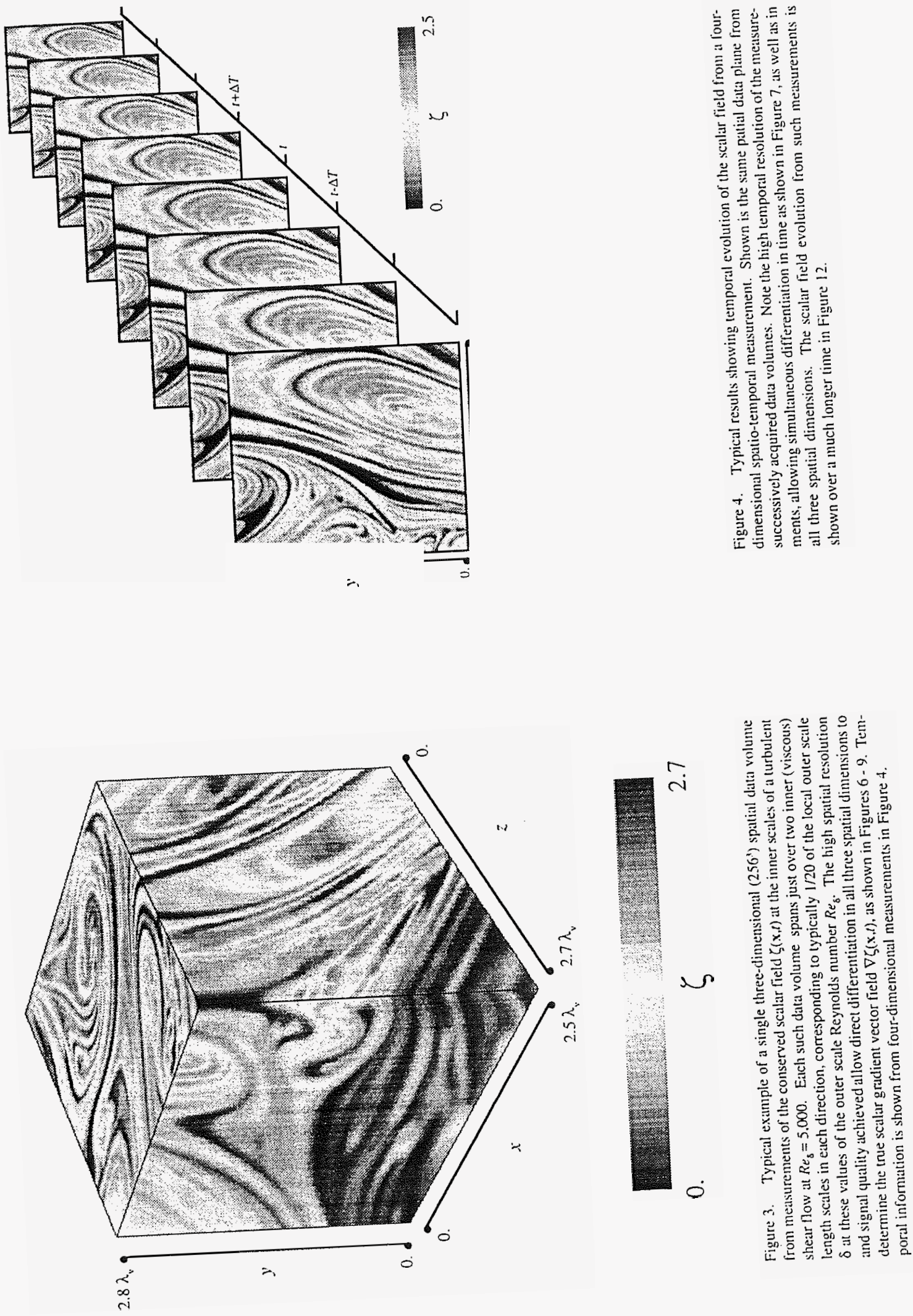
field. Fully-resolved velocity or scalar field measurements thus need to nieet these much more stringent Eulerian resolution requirements. Note that these can be related to the local inner time scale as $\tau_{v}=A T_{v} \cdot R e_{\delta}^{1 / 4}$, and to the local outer time scale as $\tau_{\delta}$ $=\left(T_{v} / \Lambda\right) \cdot R e_{\delta}^{3 / 4}$.

Finally, note that statistics of velocity and scalar fields obtained from time-series measurements will converge on the outer time scale $(\delta / u)$, while velocity gradient and scalar gradient statistics convergeon the advective time scale $T_{v}$ or $T_{\mathrm{D}}$ for Eulerian time-series measurements, and on the inner length scale $\lambda_{v}$ or $\lambda_{\mathrm{D}}$ for spatial measurements.

\section{Fully-Resolved vs. Over-Resolved Measurements}

Fully-resolved scalar field measurements in turbulent flows thus require ut least Nyquist sampling relative to $\lambda_{D}$ in space and relative to $T_{\mathrm{D}}$ in time. Velocity field measurements similarly require Nyquist sampling relative to $\lambda_{v}$ and $T_{v}$. This level of resolution allows accurate differentiation in space and time to permit determination of the scalar gradient vector field $\nabla \zeta(\mathrm{x}, t)$ and the velocity gradient tensor field $\nabla \mathbf{u}(\mathbf{x}, t)$.

Assessing if a given set of experimental data are fully resolved can be done via a procedure analogous to "grid convergence" assessments in numerical simulations. The dissipation field $\nabla f \cdot \nabla f(\mathrm{x}, t)$ associated with the energy $f^{2}(\mathrm{x}, t)$ of any measured quantity $f(\mathrm{x}, t)$ can be integrated over the measurement domain. with the procedure repeated as the resolution in the measured data $f(\mathbf{x}, t)$ is effectively degraded (post hoc) by successive averaging over adjacent points. When the result approaches a resolution-independent value, then the data are fully-resolved. Application of this procedure to the present scalar field measurements is demonstrated in the following section.

While the limits above set the minimum resolution required For fully-resolved measurements, it is noteworthy that much higher spatial or temporal resolution is not always desirable. Since data are discretized not only in space and time, but also in digital signal level, it is apparent that there is a finest resolution limit beyond which adjacent points will take on in the same digital signal level, and thus compromiscdifferentiability of the data. For any field $f(\mathrm{x}, t)$, the finest spatial resolution Ax and temporal resoltition $\Delta t$ occur at critical values of the parameters

$$
B_{1} \equiv \frac{|\nabla f|}{\Delta f / \Delta x} \quad \text { and } \quad B_{1} \equiv \frac{|\nabla f|}{\Delta f /(u \cdot \Delta t)} \text {, }
$$

where $|\nabla f|$ characterizes the local gradient magnitude, and $\Delta f$ is tlic difference in $f$ between successive digital signal levels. When $B$ becomes sufficiently small. spatially or temporally adjacent points will be at the same digital signal level, contributing to an underestimate in the magnitude of the gradient field $\nabla f(\mathrm{x}, t)$ or the time derivative $\partial f / \partial t(\mathrm{x}, t)$, and affecting the orientation of the gradient vector.

\section{FOUR-DIMENSIONAL SCALAR FIELD MEASUREMENTS}

Three- and four-dimensional measurements of the universal small-scale structure of conserved scalar mixing in turbulent flows have become possible over the past few years (Dahm, Southerland \& Buch 1991; Buch \& Dahm 1996a; Southerland \& Dahm 1994, 1996). This section describes such measurements having spatial resoltition finer than the scalar diffusion length scale $\lambda_{\mathrm{D}}$ and temporal resoltition finer than the scalar advection time scale $T_{\mathrm{D}}$, and with results spanning up to $2.5 \lambda_{v}$ in each spatial dimension and I.2 $\tau_{\delta}$ in the temporal dimension. The resulting conserved scalar field data $\zeta(\mathrm{x}, t)$ simultaneously span all three spatial dimensions and time, and have sufficiently high signal quality to accurately determine the true scalar gradient vector field $\nabla \zeta(\mathrm{x}, t)$. The measurements presented here were made at a fixed location in the self-similar far field of an axisymmetric turbulent jet at outer-scale Reynolds numbers $R e$, in the range 2,600 - 5,000 and with Taylor-scale Reynolds numbers $R e_{\lambda}$ ranging from 38 to 52 .

\section{Scalar Field Measurement Technique}

The measurements are based on high-speed imaging of the laser induced fluorescence intensity emitted by the concentration field of a passive water-soluble dye having $S_{C} \approx 2075$, which mixes with undyed fluid in a turbulent shear flow. A steady axisymmetric turbulent jet was formed by issuing a weak aqueous disodium fluorescein solution through a $4.9 \mathrm{~mm}$ axisymmetric nozzle into de-ionized water in a tank. All measurements were made 235 diarnetersdownstream of the jet exit $(x=1.15 \mathrm{~m})$, for which the resulting $\delta(x)=50 \mathrm{~cm}$. For comparison, the threedimensional measurement volume was typically $2.5 \mathrm{~cm}$ on each side. The concentration field $\zeta(\mathbf{x}, t)$ was measured repeatedly in time at as many as $256^{3}$ points within a small three-dimensional spatial volume located 26 diameters $(13 \mathrm{~cm})$ off the jet centerline. A highly collimated beam from a $5 \mathrm{~W}$ argon-ion laser operated in multi-line emission mode was swept in a raster fashion through this volume, and the resulting laser induced fluorescence from dye-containing fluid was imaged onto a high-speed, planar, $256 x 256$ clement. photodiode array (EG\&G Rcticon MC9256/MB9000) by a Vivatar $100 \mathrm{~mm} f-2.8$ macro lens operated at full aperture with an orange Mie filter. Figure 2 shows key elements of the data acquisition system assembled to convert and store the serial output from the photodiodearray in 8-bit digital format. The array formatter provided a non-interlaced, sampled-and-held output train to the A/D converter. An extenial clock signal drove the array at pixel rates up to $1 \mathrm{I} \mathrm{MHz}$, corresponding to a framing rate up to 120 frames per second. A dualported image processor effectively acted as a high speed $16 \mathrm{MB}$ buffer in which segments of the array output data stream were temporarily stored en route to four 823.9 MB capacity disk drives. The 3. I GB capacity of the disks allowed continuous interleaved acquisition of nearly 200 individual $256^{3}$ spatial data volumes, or over 50.000 individual $256^{2}$ data planes at the sustained throughput rate of $9.3 \mathrm{MB} / \mathrm{sec}$. The resulting measured fluorescence intensity field $F(\mathrm{x}, t)$ was subsequently converted to the true dye concentration field $c(\mathrm{x}, t)$ via the full multiline form of Beer's Law, and then to the conserved scalar field $\zeta(x, t)$ as described in Southerland \& Dahrn $(1994,1996)$.

Each mcasuremcnt produces tlic scalar field at over 3 billion individual points in space and time, structured as shown in Figure 1. To estimate the resulting spatial and temporal resolution, note that the local outer scale $\delta(x) \approx 0.44 . x$ and centerlinc veloc- 

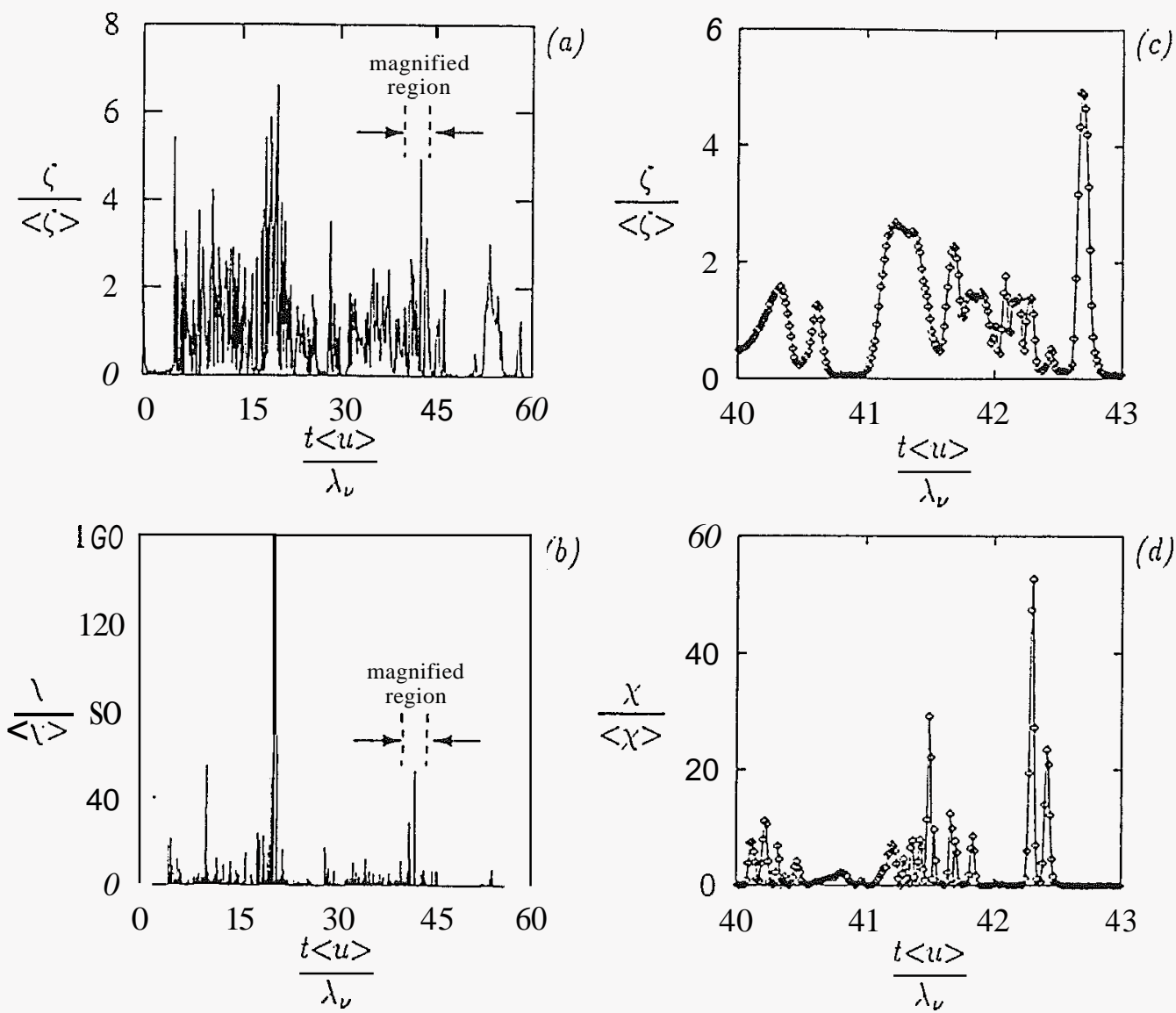

Figure 5. Example time series results from the present fully-resolved. four-dimensional, spatio-temporal measurements, showing time-varying conserved scalar values $\zeta(x, l)$ at a single spatial data point at lop. aridthe scalar energy dissipation values $\nabla \zeta . \nabla \zeta(x, l)$ ai a single spatial data point al bot tom. The entire durition of the measurement is shown ai left. as well as a magnified section at right demonstrating the high temporal resolution achieved. Symbols show the discrete nteisured values.

ity $u(x)=7.2(J / \rho)^{1 / 2} \cdot x^{-1}$, with $/$ the jet source momentum tlux and $\rho$ the ambient fluid density. For example, at the outer scale Reynolds number $R e, \equiv(u \delta / N)=3,700$ and with the Schmidt number of 2075, the local strain-limited molecular diffusion length scale estimate is $\lambda_{\mathrm{D}}=257 \mu \mathrm{m}$ and the local advection time scale estimate is $T_{\mathrm{D}} \approx 103 \mathrm{msec}$. For comparison, the in-plane spatial resolution was $\Delta(x, y)=109 \mu \mathrm{m}$. The $(1 / e)$ laser beam thickness was measured as IS I $\mu \mathrm{m}$. Deconvolution of the scalar field measurements among adjacent planes increases the effective spatial resolution in the $z$-direction to the interplane separation $\mathrm{Az}=120 \mu \mathrm{m}$. These values show that both the characteristic scale of the pixel image volume $(\Delta x \cdot \Delta y \cdot \Delta z)^{1 / 3}$ and its maximum dimension $(\mathrm{Az})$ are less than $0.5 \lambda_{\mathrm{D}}$. Similarly. the temporal separation between successive data planes was $\Delta t=S .9$ $\mathrm{msec}$. and comparing with the diffusion scale advection time of $103 \mathrm{msec}$ verifies that the present measurements resolve essentially all of the fine scale structure of the local turbulent mixing process.

Figure 3 gives a representative example of a single threedimensional $256^{3}$ spatial data volume from such a measurement.
In this case. the outer-scale Reynolds number $\boldsymbol{R} \boldsymbol{e}$, is 5.000. and the data volume gives an indication of the access to threedimensional fine scale structure accessible by such meisurements. Similarly. representative results from four-dimensional data are given in Figure 4. which shows a time serics of the scalar field in the same spatial data plane from eight temporally successive spatial data volumes, and in Figure 5.

In terms of classical Kolrnogorov variables. for the fourdimensional data, the intervolume time of $53 \mathrm{msec}$ is typically less than half the scalar diffusion scale advection time $\lambda_{\sigma} / u$ and thus entirely negligible in comparison with the Kolmogorov time scale $(v / \varepsilon)^{1 / 2}$. Similarly, the interplane time is $8.9 \mathrm{msec}$, which even in the worst case is less than $1 / 10 \lambda_{\sigma} / u$, and thus is entirely trivial in terms of the Kolmogorov time. Consequently, the data acquisition rate is sufficient to cffectively treeze the scalar field. and to entirely freeze the underlying velocity field.

The high spatial and temporal resolution achieved, together with the high signal quality attained. allows accurate diflerentiation of the measured conserved scalar ficld in all three spatial di- 


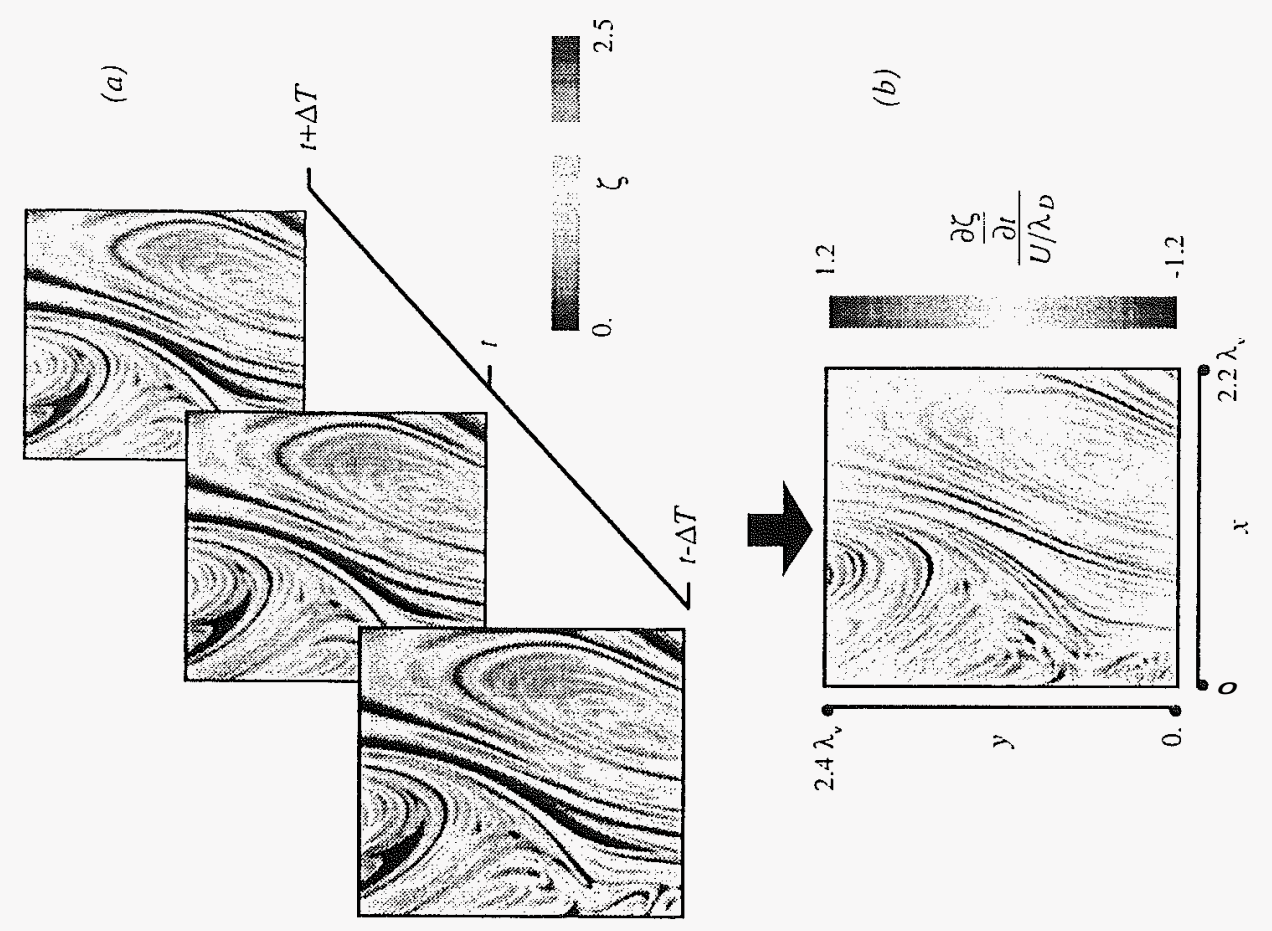

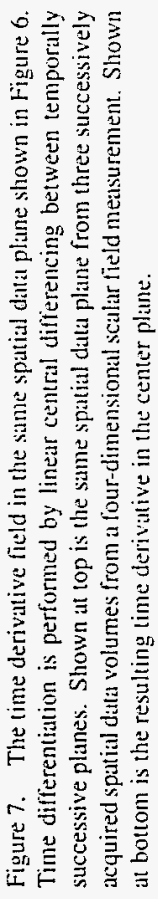

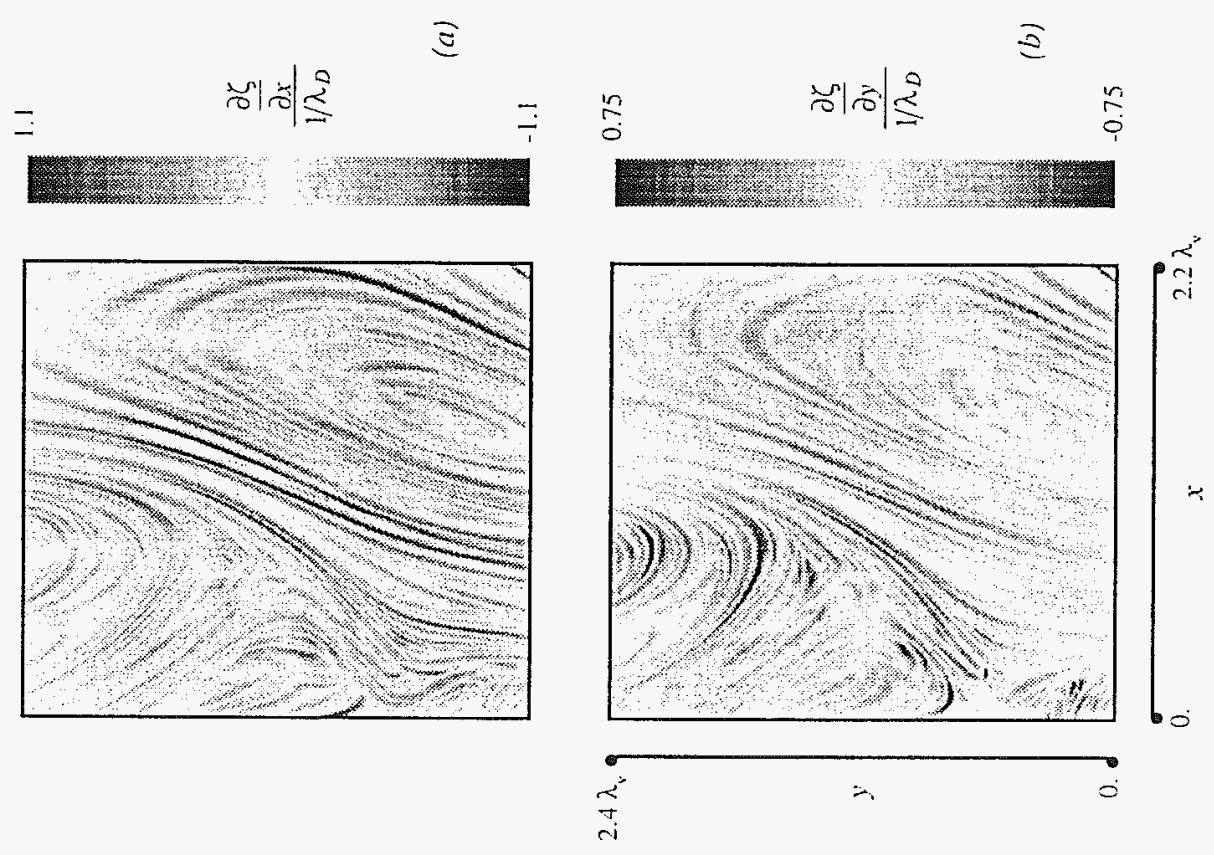

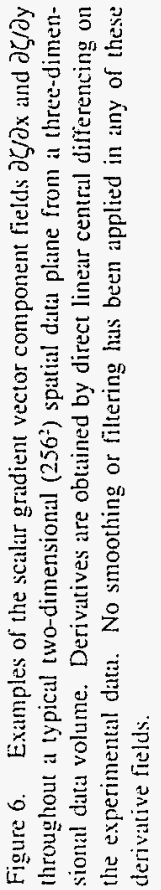



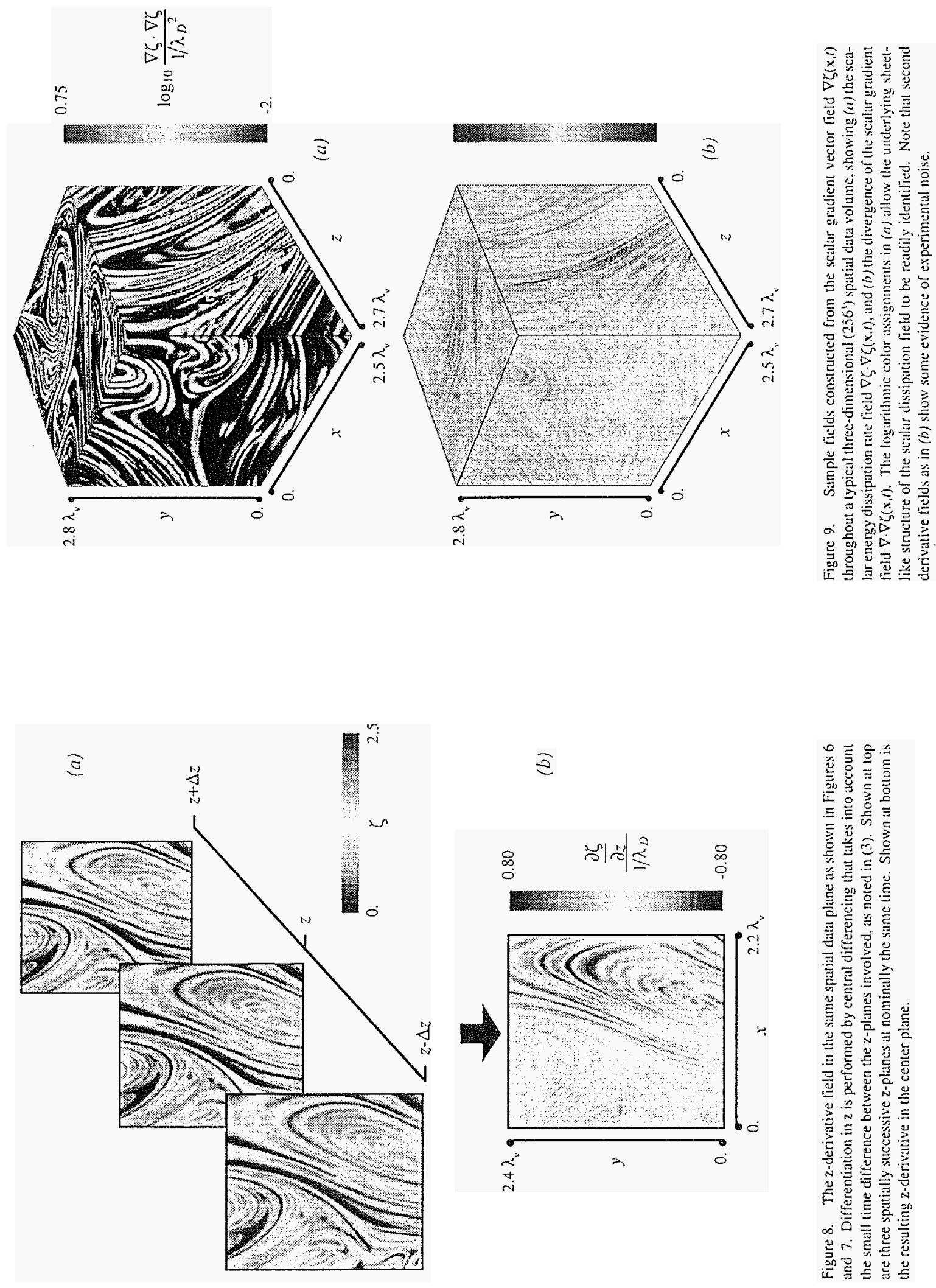
mensions and in time. as shown in Figures 6-9. This makes it possible to determine the components of the true local instantaneous scalar gradient vector field $\nabla \zeta(x, t)$ throughout these three- and four-dimensional data. without any need to resort to various approximations based on Taylor's hypothesis as is commonly required. This in turn permits determination of the true scalar energy dissipation rate field $\nabla \zeta \cdot \nabla \zeta(\mathbf{x}, t)$, and thus an assessment of the geometric scaling properties of the highly compact support on which this field is concentrated in turbulent sheir tlows.

Similarly, the temporal separation between adjacent data planes within each three-dimensional (2563) spatial data volume. and between the same data point in successive spatial volumes with fewer z-planes in the fully four-dimensional data. is shorter that the local diffusion scale advection time $\tau_{0}$. As a result it is possible to extract fully-resolved time series data of the type shown in Figure 5 from both the conserved scalar field $\zeta(x, l)$ and scalar energy dissipation rate field $\nabla \zeta \cdot \nabla \zeta(\mathrm{x}, \imath)$.

Differentiation of such data in $x, y$, and $t$ is straightforward via linear central difference operators between spatially or temporally adjacentplanes, as shown in Figures 6 and 7. Note that all derivative results are shown without any smoothing or filtering. Moreover, with four-dimensional data it is even possible to take into account the small time differenceover which spatially adjicent planes are acquired when differentiating in $\mathrm{z}$ as

$$
\begin{gathered}
\frac{\partial \zeta}{\partial z} \equiv \frac{1}{2 \Delta z}[\zeta(z+\Delta z t+\Delta l)-\zeta(z-\Delta z \cdot t-\Delta t) \\
\left.-2 \frac{\partial \zeta}{\partial t}(z . t)\right]+O\left(\Delta^{2}\right) .
\end{gathered}
$$

This avoids errors introduced by naive npplication of the direct central difference. and determines all three components of the gradient vector field $\nabla \zeta(x, I)$ with equivalent levels ot'accuracy.

Figure $9 a$ shows an example of the instantaneous scallil energy dissipation rate field $\nabla \zeta \nabla \zeta(x, l)$ throughout such a ilirecedimensional (2563) spatial data volume. and Figure $9 b$ gives the resultingdivergence of the scallar gradicnt field $\nabla, \nabla \zeta(x, l)$ in the same spatial data volume. Figure 10 shows the result obtilined when the experimental "grid convergence"procedure described in the previous section is applied to tlie present four-dimensional scalar field data. This shows that the present resolution levelessentially reaches the knee in curve. with approximately $80 \%$ of the scalar energy dissipation captured by the present measurements. A factor of ten finer resolution would be needed to calpture $98 \%$ of the dissipation: a factor of three coarser resolution would capture less than $15 \%$ of the total dissipation.

Note that the imaged region in the turbulent scalar ficld in these experiments typically spans less than $1 / 15$ of the local outer scale $\delta$, and is comparable to the local inner scale $\lambda_{v}$ of the flow. The structure of velocity and scalar fields in turbulent

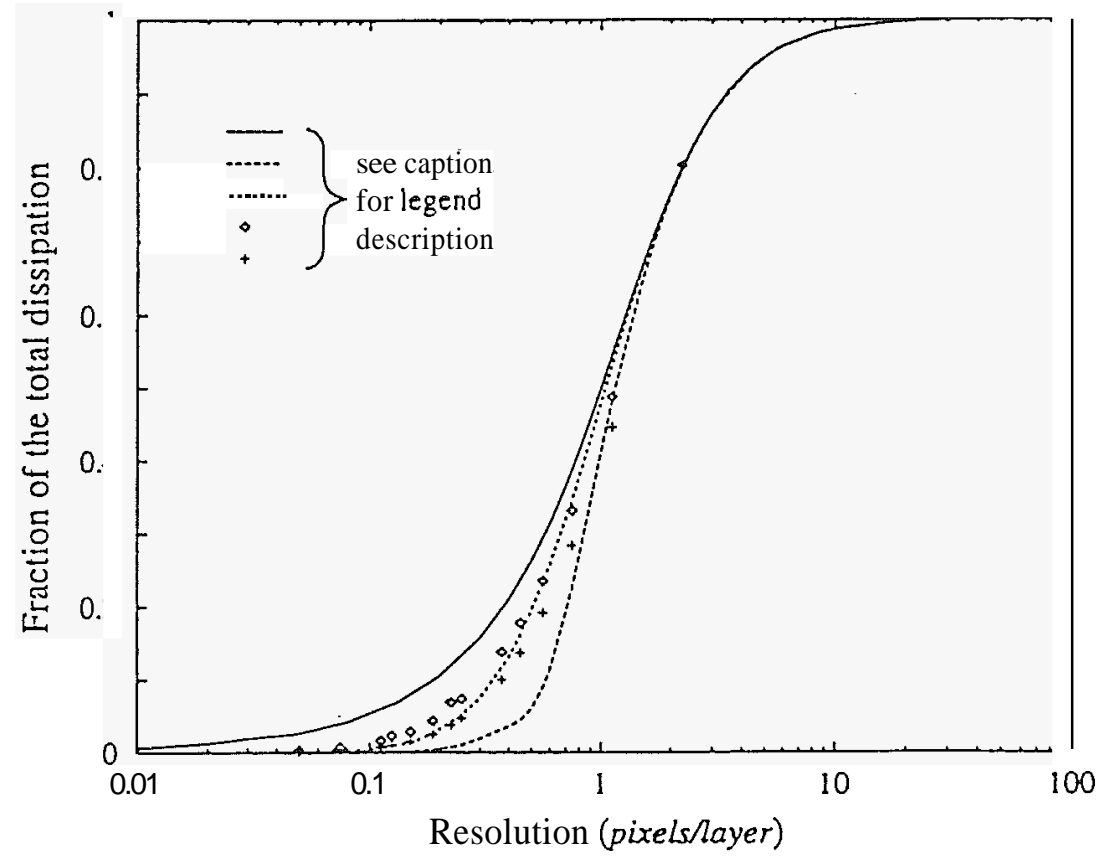

Figure 10. Experimental grid convergence test of the true resolution achieved in these measuremeits, showing the fraction of the total scalar dissipation $\nabla \zeta . \nabla \zeta(x, l)$ captured by a measurement having any given number of pixels per inner scale $\lambda_{\mathrm{D}}$. The solid line is the theoretical result for a single disipaition layer. The shon dished line is the theoretical result for a set of parallel interacting layers having the sitme distributions of scalar endpoints ind latyer icparations as the experimental data. Note the good agreement with the latter. indicating that the present measurements resolve $80 \%$ of the total dissigit. tion. Any measurement having resolution coarserby an order of magnitude than the present data would miss essentially all the dissipation. A factor of ten hiner resolution would be required to capture $98 \%$ of the total resolution. 
shear flows at scales near and below $\lambda_{v}$ is generally believed to be statistically universal. This contention appears to be true even for the present moderate Reynolds number flows. as evidenced by the DNS studies of Jiménez, Wray, Saffrnan \& Rogallo (1993). The estimated Taylor scale Reynolds numbers for the present data are $R e ;, \approx 45$, well within the range of values over which the DNS results of Jiménez et al showed Reynolds number independent collapse on inner variables at the smallest flow scales. Moreover. high wavenumber spatial scalar spectra from these same data (Southerland, Dahm \& Dowling 1995) show the $k^{-1}$ scaling predicted by Batchelor for large $S c$ mixing in turbulent flows. As a result, even though the present measurements are from $R e_{\delta} \approx 3,500$ turbulentjets, the fine scales seen in them are believed to be largely representative of the generic scaling properties at the inner scales of all turbulent shear flows.

\section{FULLY-RESOLVEDVELOCITY FIELI) MEASUREMENTS}

Fully-resolved, four-dimensional, spatio-temporal measurements of all three components of the velocity vector field $\mathbf{u}(\mathbf{x}, t)$, as well as all nine components of the corresponding velocity gradient tensor field $\nabla \mathbf{u}(\mathbf{x}, t)$ and associated dynamical fields obtained from it, can be obtained via the scalar imaging velocimetry (SIV) technique. This technique is based on four-dimensional scalar field measurements of the type described above, though the requirement for full spatial and temporal resolution in the scalar field, and even in the velocity field, can be relaxed as will be shown in the following section. In this section. we summarize the velocimetry technique and present sample results obtained from it. The technique is described in detail by Dahm, Su \& Southerland (1992) and by Su \& Dahm (1996a,b).

\section{The Scalar Imaging Velocimetry Technique}

In scalar imaging velocinietry, extraction of the underlying space- and time-varying velocity field froni such scalar field measurements is based on the exact conserved scalar transport eauation

$$
\left[\frac{\partial}{\partial t}+\mathbf{u} \cdot \nabla-\frac{1}{\operatorname{ReSc}} \nabla^{2}\right] \zeta(\mathbf{x}, t)=0 .
$$

Given fully space- and time-differentiable scalar field data $\zeta(\mathrm{x}, t)$, the only unknowns in this equation are the components of the velocity field $\mathbf{u}(\mathrm{x}, t)$. As noted in the Introduction, it is possible to extract the velocity field despite the fact that only the local component of $\mathbf{u}$ along the scalar gradient vector direction $\hat{e}_{\nabla \zeta}$ affects the scalar field evolution. This is done by making use of additional constraints, such as the smoothness condition that relates the finest length scales admissible in the velocity and scalar gradient vector fields in turbulent flows, as was pointed out by Dahm, Su \& Southerland (I 992). Such constraints must he imposed in all single-scalarbased velocimetry methods to obtain the velocity field and thereby exclude the spurious additive fields admitted by (4), which must have streamlines confined to isoscalar surfaces. It is preferable that these constraints be physically-based and imposed explicitly, as is clone here, so that it is clear that the truc velocity field $\mathbf{u}(\mathbf{x}, t)$ is obtained. In the correlation-based approach (c.g. Maas 1993; Merkelet al 1995; Tokamaru \& Dimotakis 1995) the constraints are imposed im- plicitly by the correlation template in the numerical method used in the inversion and has no direct physical basis, in which case the relevance of the resulting fields to the true velocity field may be unclear. In the SIV technique, the constraints are physicallybased but applicable only in turbulent flows and thus, unlike LDV, PTV or PIV, the method can only be applied in turbulent flows.

In the SIV method, there are two closely related approaches by which this inversion can be accomplished. The first, referred to as direct inversion scalar imaging velocimetry, involves inverting (4) directly throughout the four-dimensional data space to obtain the velocity component field $\iota_{\|}(\mathrm{x}, 1)$ which lies along the scalar gradient vector direction $\hat{e}_{\nabla \zeta}(\mathrm{x}, t)$. The full vector field is then obtained by imposing the smoothness constraint on $\nabla u_{\|}(\mathbf{x}, t)$ to yield an iterative procedure that converges to the true velocity field $\mathbf{u}(\mathbf{x}, t)$, as described by Dahm. Su \& Southerland (1992). The second approach, referred to as integral minimization scalar imaging velocimetry, recognizes that any measurementerrors in the scalar fielddata $\zeta(\mathrm{x}, t)$ will lead to a non-zero right hand side in (4). The method thus instead determines the velocity field that minimizes an integral of a functional $E$ composed of the right hand side of (4) together with the smoothness constraint, and possibly other physical constraints if desired. Thus

$$
E \equiv E,+\alpha^{2} E_{2}+\beta^{2} E_{3}+\cdots
$$

where

$$
\begin{gathered}
E_{1} \equiv \| \frac{\partial}{-}+\mathbf{u} \cdot \nabla- \\
E_{2} \equiv(\nabla \cdot \mathbf{u}(\mathbf{x}, t))^{2},
\end{gathered}
$$

and

$$
E, \equiv \nabla \mathbf{u}: \nabla \mathbf{u}(\mathbf{x}, t)
$$

where $E$, is the smoothness constraint and $E_{2}$ allows the continuity constraint to be included. The factors $\alpha^{2}, \beta^{2}, \ldots>0$ allow control over the relative weights assigned to each of the $E_{i}$. By selecting the weight assigned to the smoothness constraint, the correct ratio of length scales in the velocity and scalar fields can be ensured.

Note that all the $E_{i} \geq 0$ were chosen to be second-order in $u$, so that fast, linear iterative methods can be used to perform the minimization. Moreover these constraints were chosen so that the resulting system contains only first- and second-ordcr derivatives of the velocity components, for which compact discrete difference operators lead to a sparse linear system. The resulting smoothness constraint in (8) is in the form of a regularizing operator, which arc commonly used in inverse problems. The mathematical basis of such operators is described by Tikhonov \& Arsenin (1977); operators of the form of $E$, are known as Tikhonov stabilizers of first order.

For $E$ as defined here. including only first derivatives of the dependent variables $u_{i}$, the Euler characteristic equation that minimizes the integral of $E$ over the domain has the general form

$$
\frac{\partial E}{\partial u_{i}}-\sum_{j=1}^{n} \frac{\partial}{\partial x_{j}}\left(\frac{\partial E}{\partial\left(\partial u_{i} / \partial x_{j}\right)}\right)=0
$$




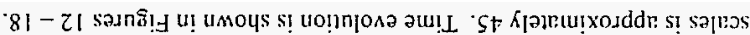

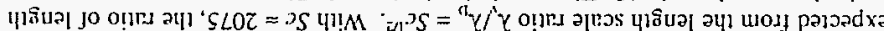

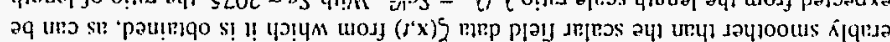

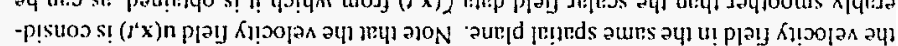

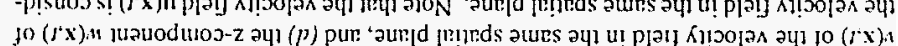

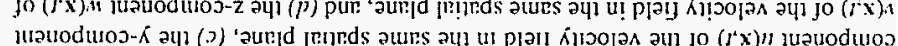
manod usos- $\alpha$ aा

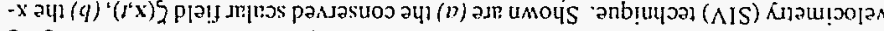

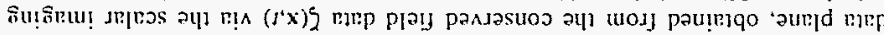
10. -qo sp pr

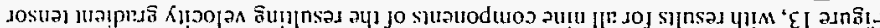

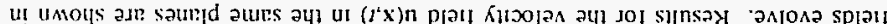

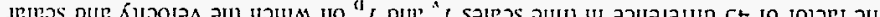
या

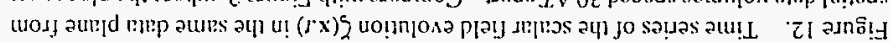

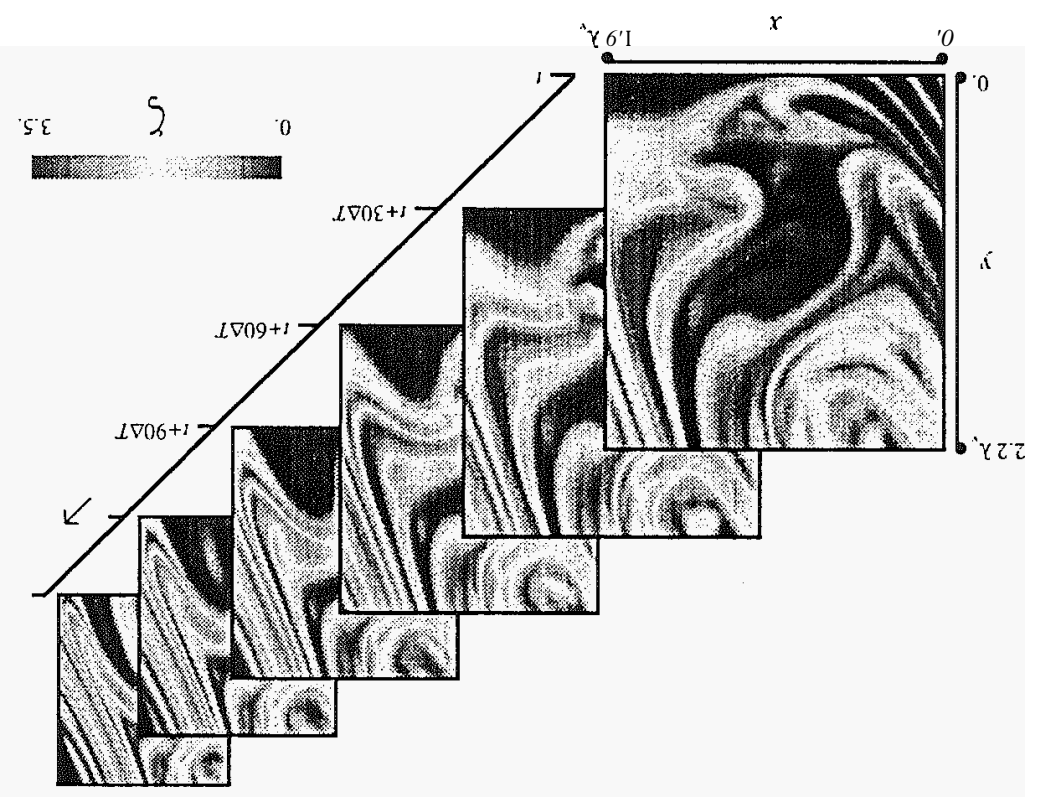

(p)

$$
\cdot \bar{c}
$$

L $\quad(n \gamma / \Lambda) / n$

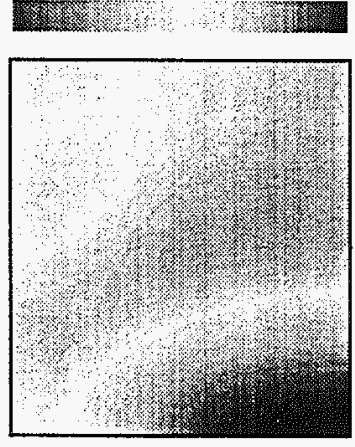

$\angle 1$

$(\wedge \chi / \wedge) / n$

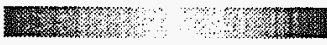

(q)

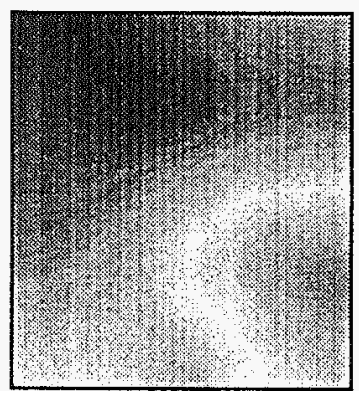

(J)

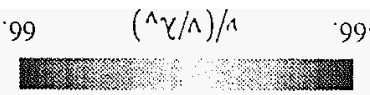

2\%

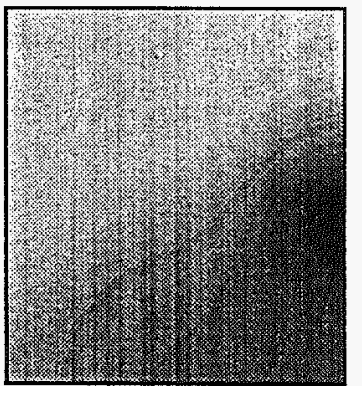

(v)

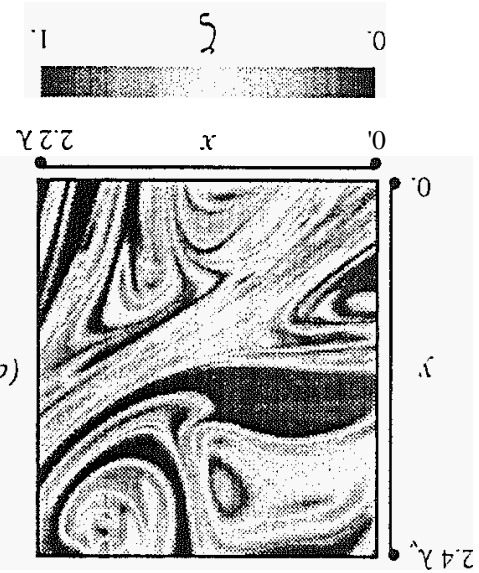




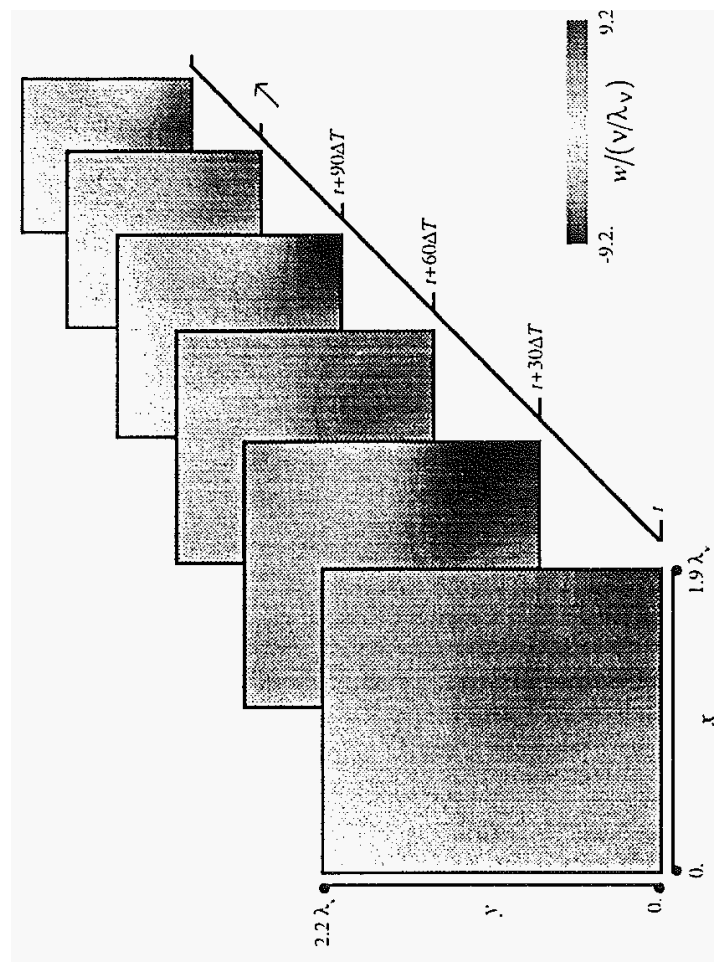

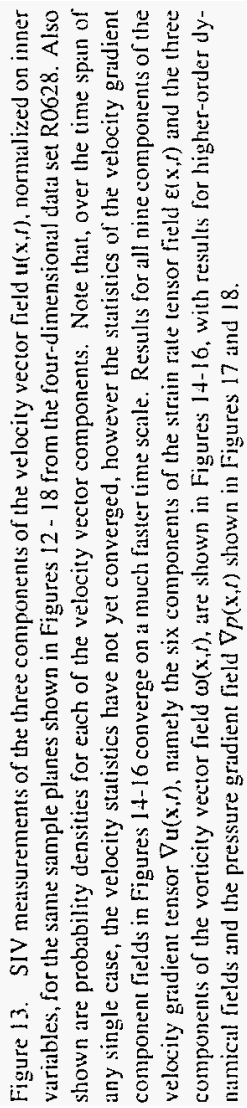
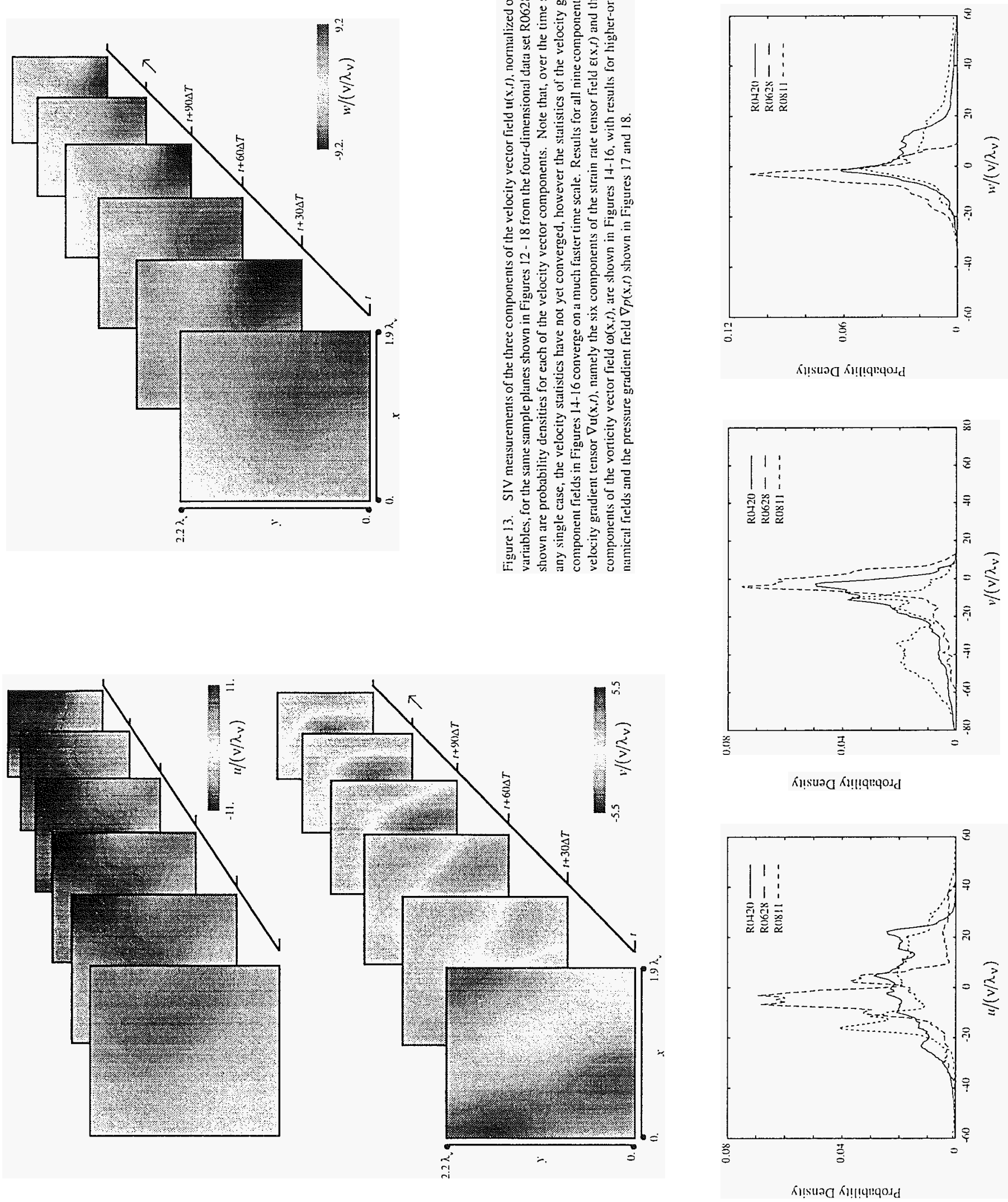


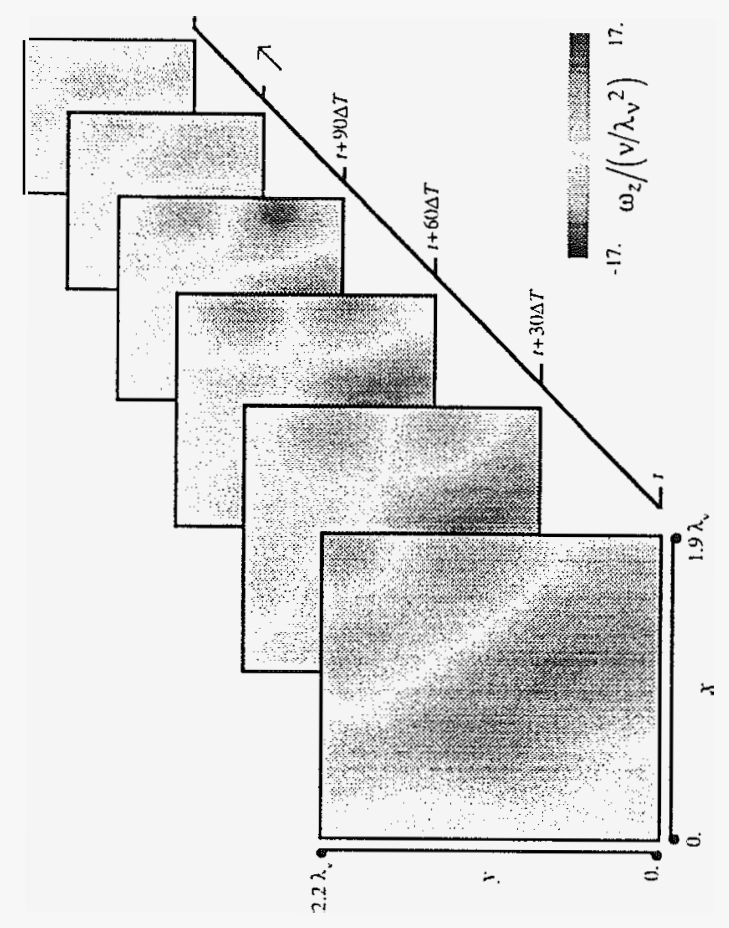

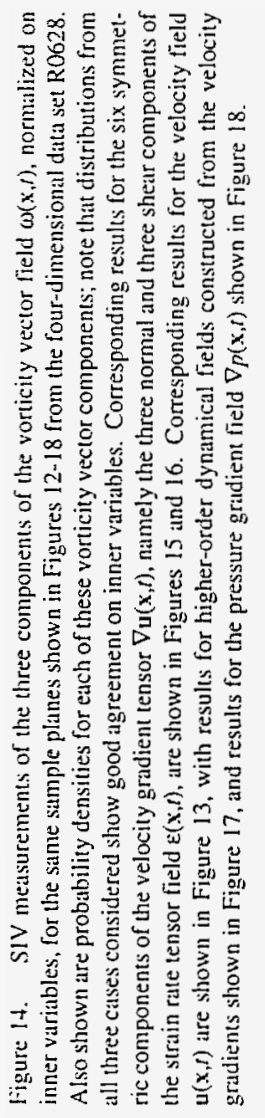
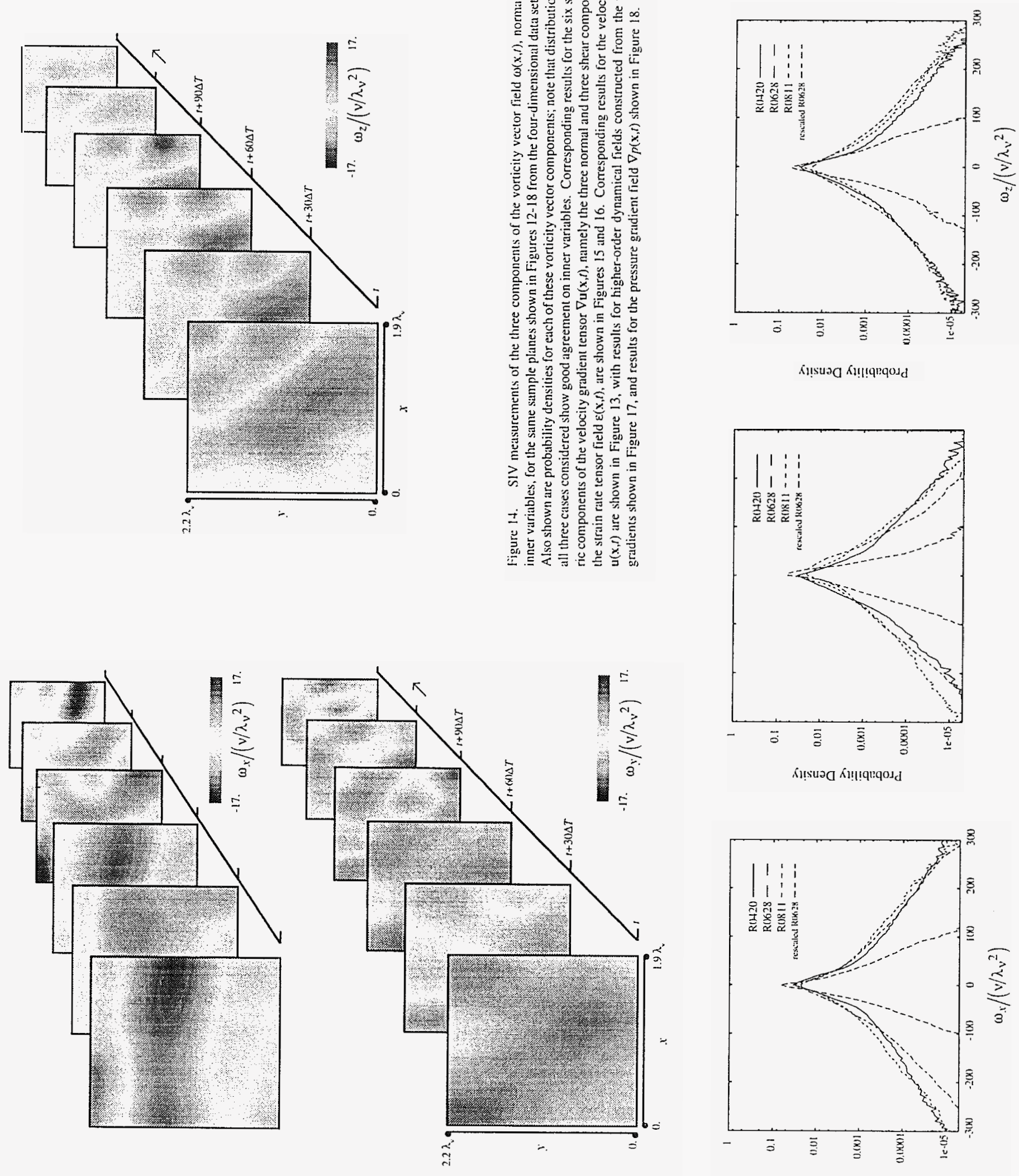

K!!surg K!!!qnqoud
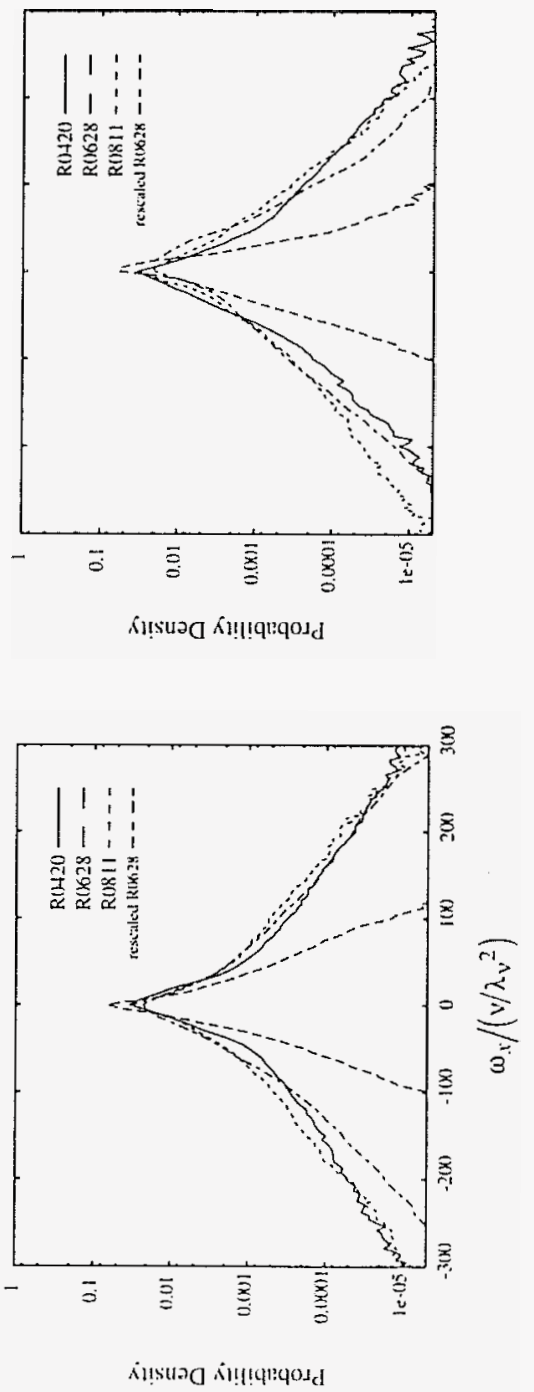

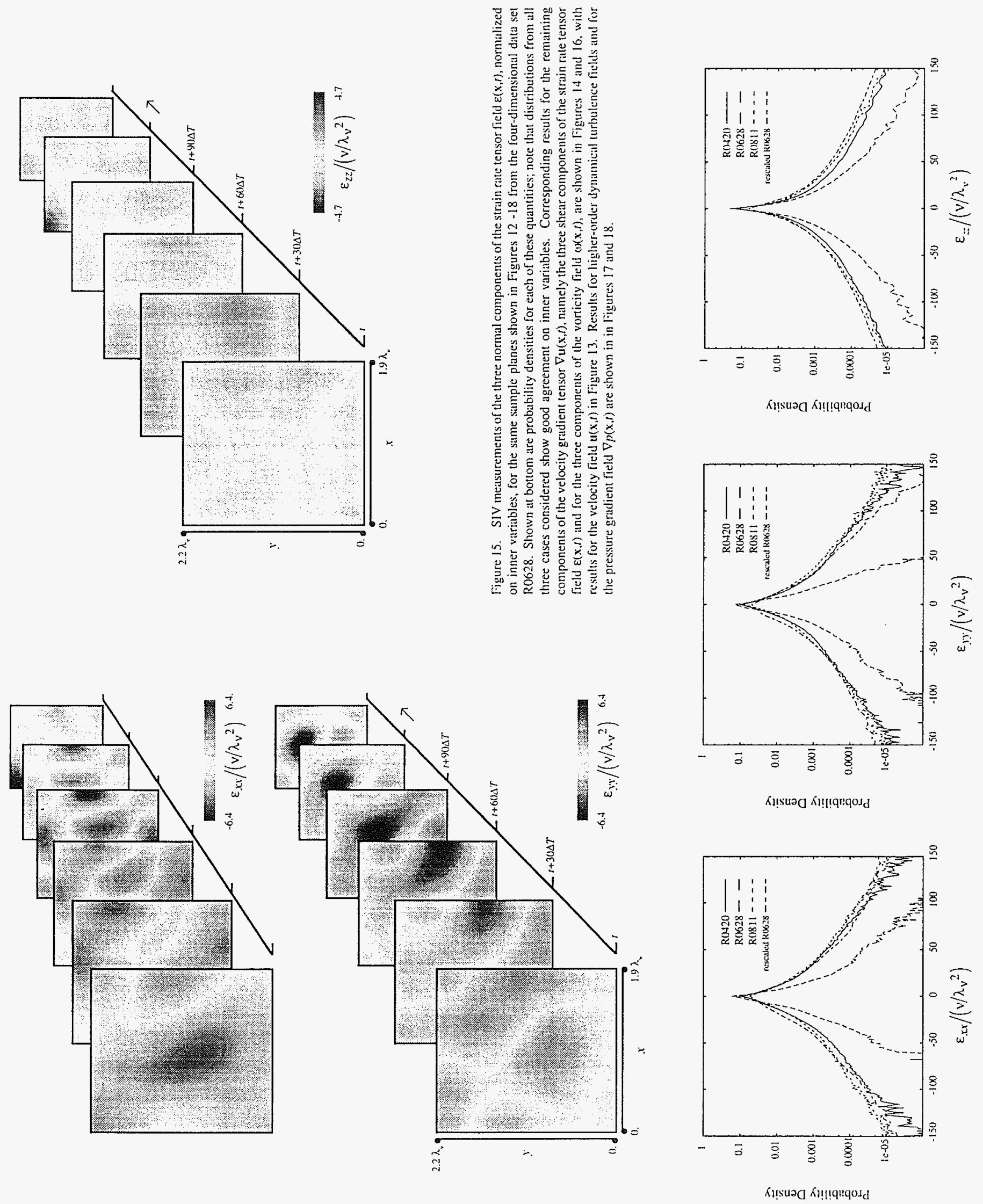
$\left(i^{n} \times / n\right) / p^{\prime \prime} 3$

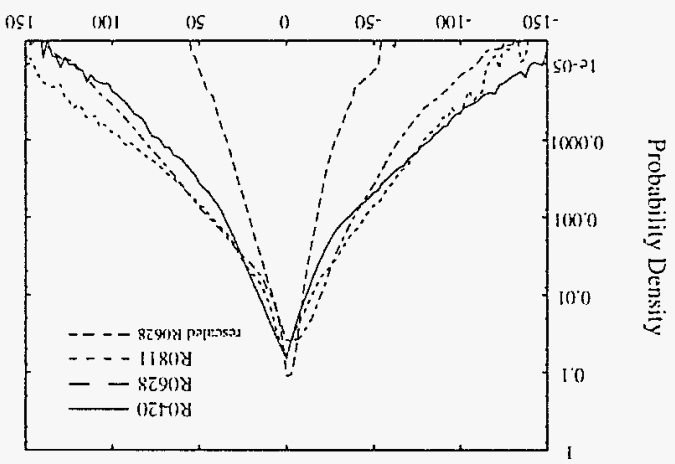

$\left(i^{\wedge} \gamma / \Lambda\right) / i_{3}$

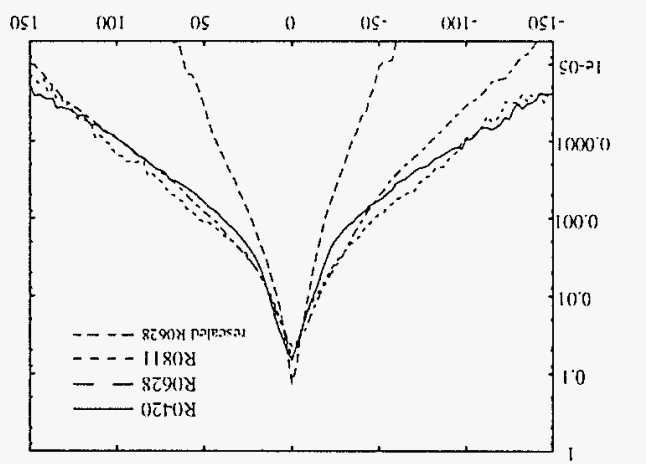

$\left(\tau^{\wedge} \gamma / n\right) / \dot{u}_{3}$

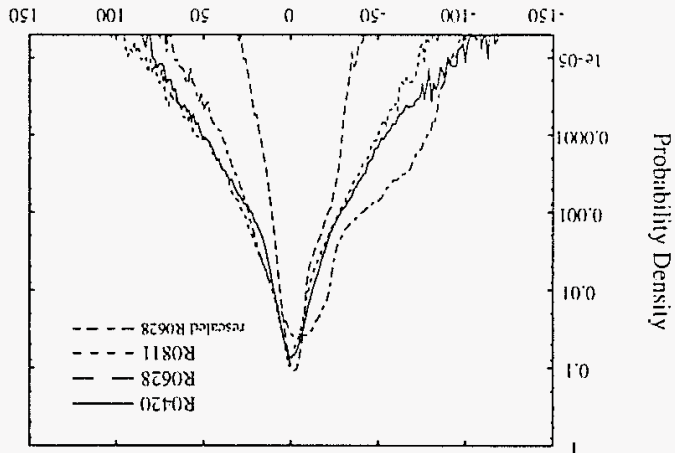

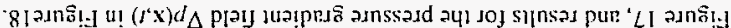

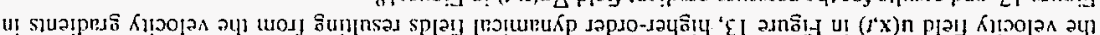

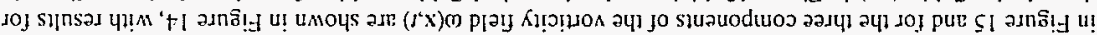

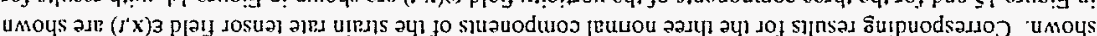

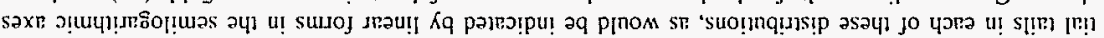

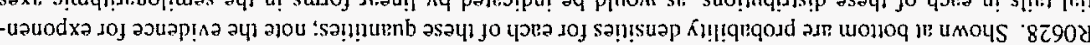

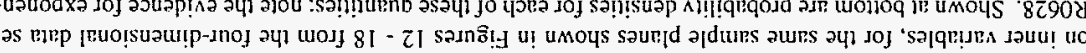

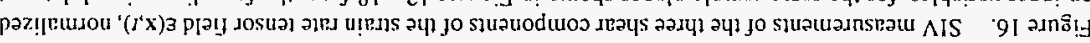

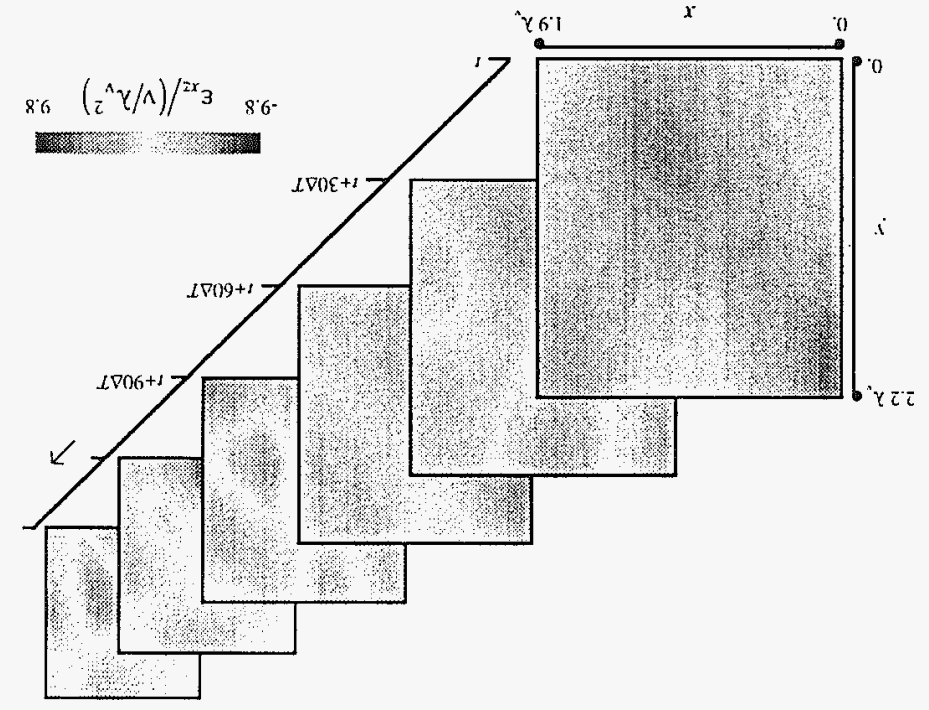

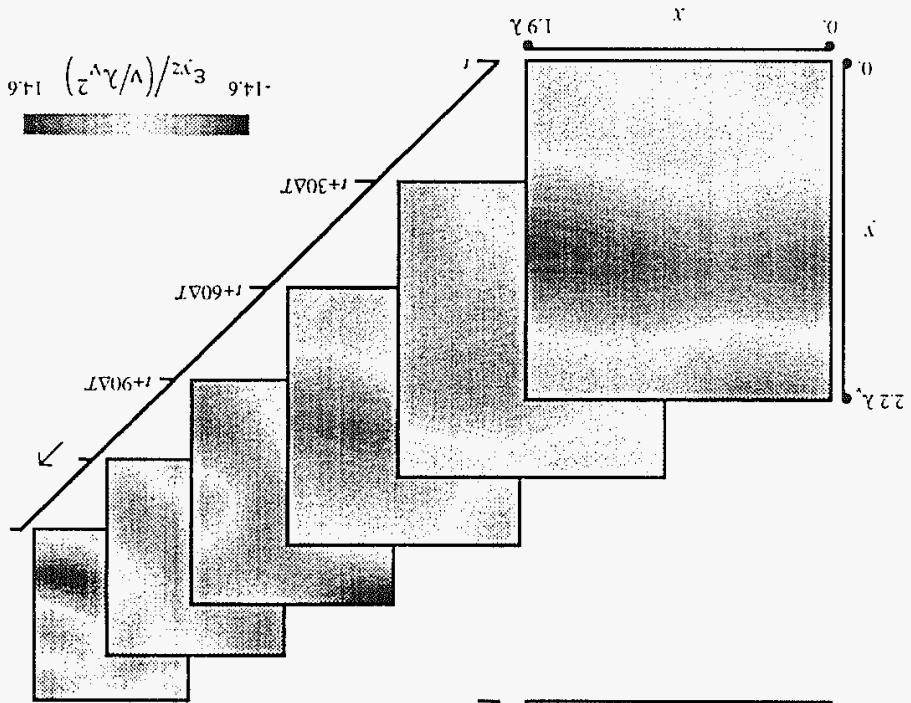

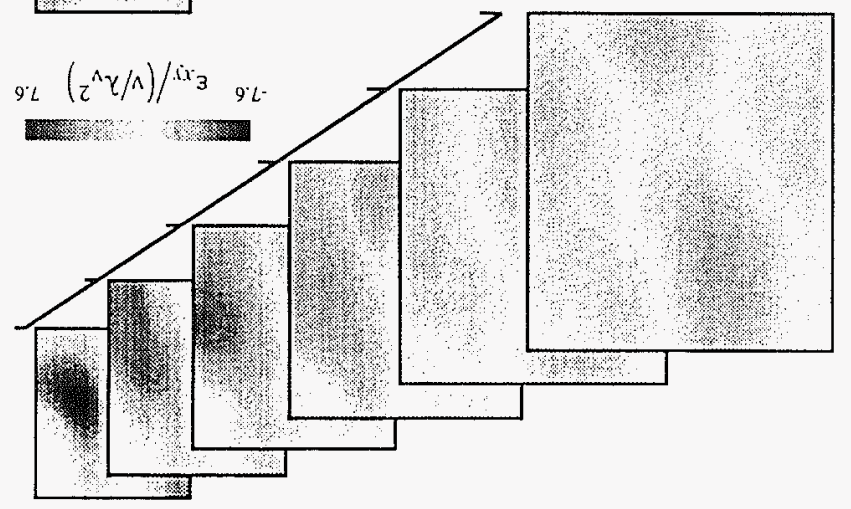


$\left(i^{\wedge} \gamma / \Lambda\right) /(m \cdot 3 \cdot m$

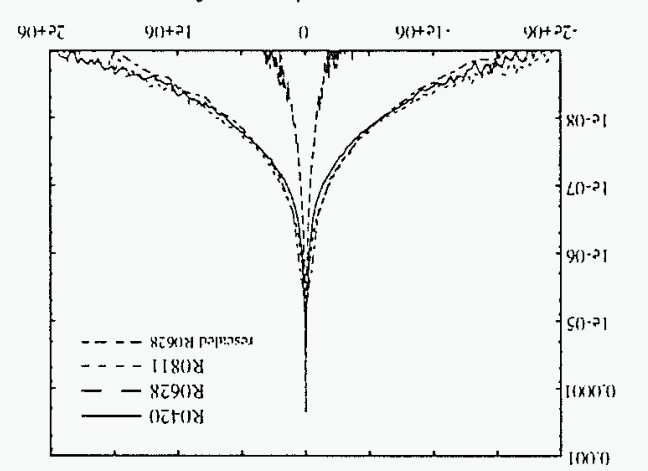

$\left(i^{\wedge} \gamma / n\right) / m \cdot m \frac{z}{i}$

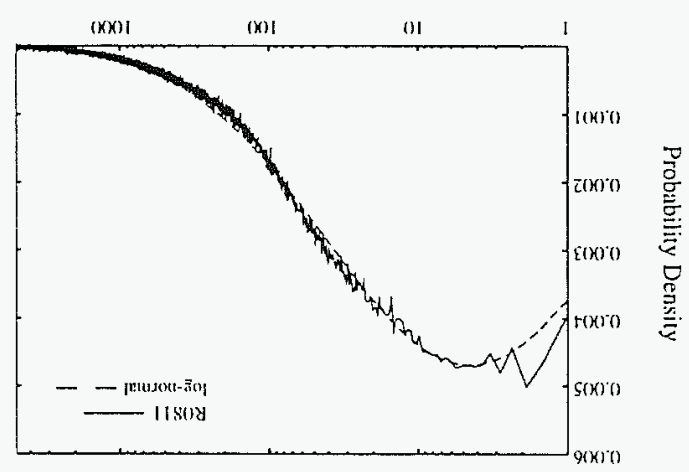

$z^{n}\left(\bar{\imath}^{\wedge} \gamma / \Lambda\right) / \Phi$

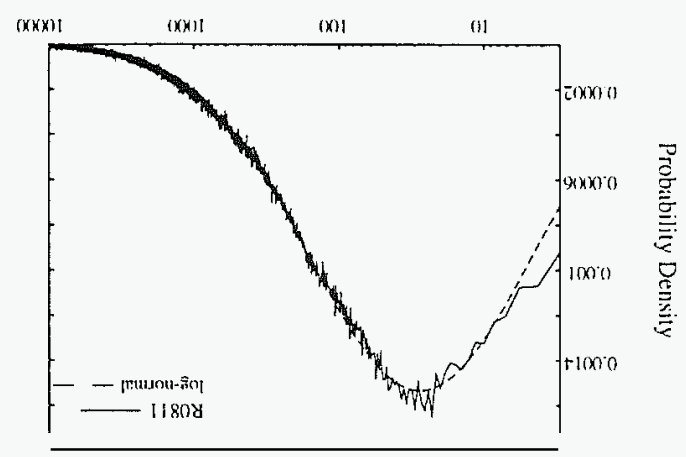

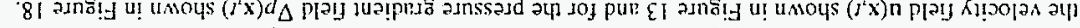
Jol Sins.

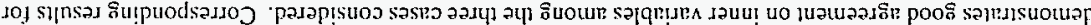

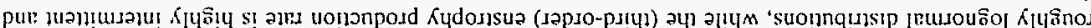

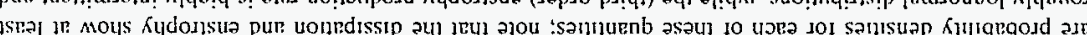
's

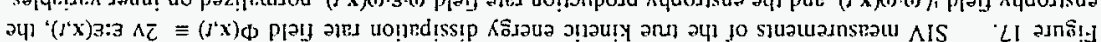

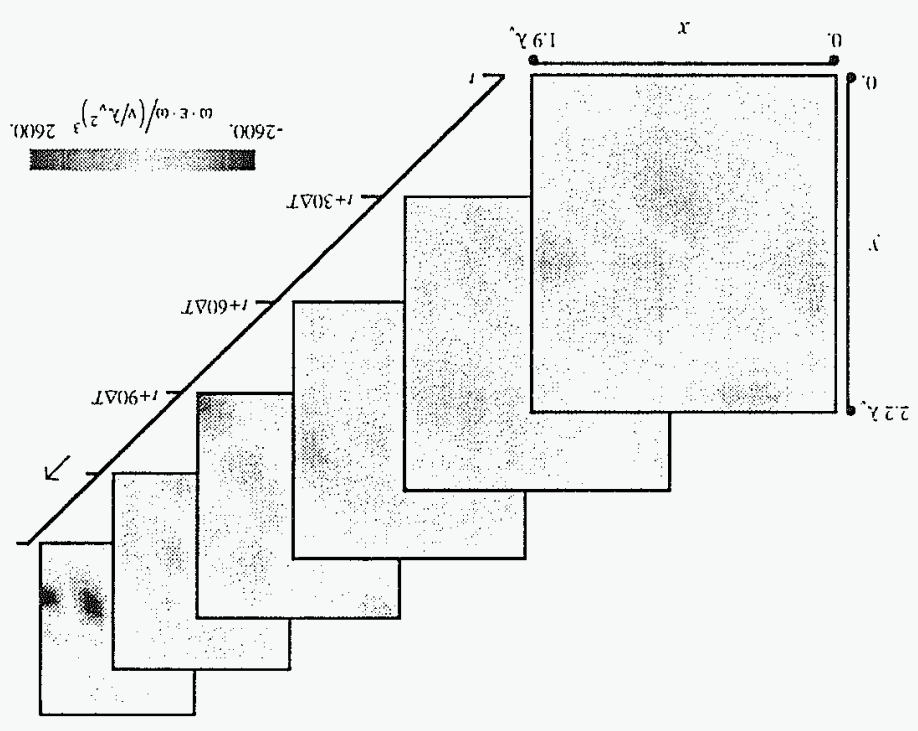

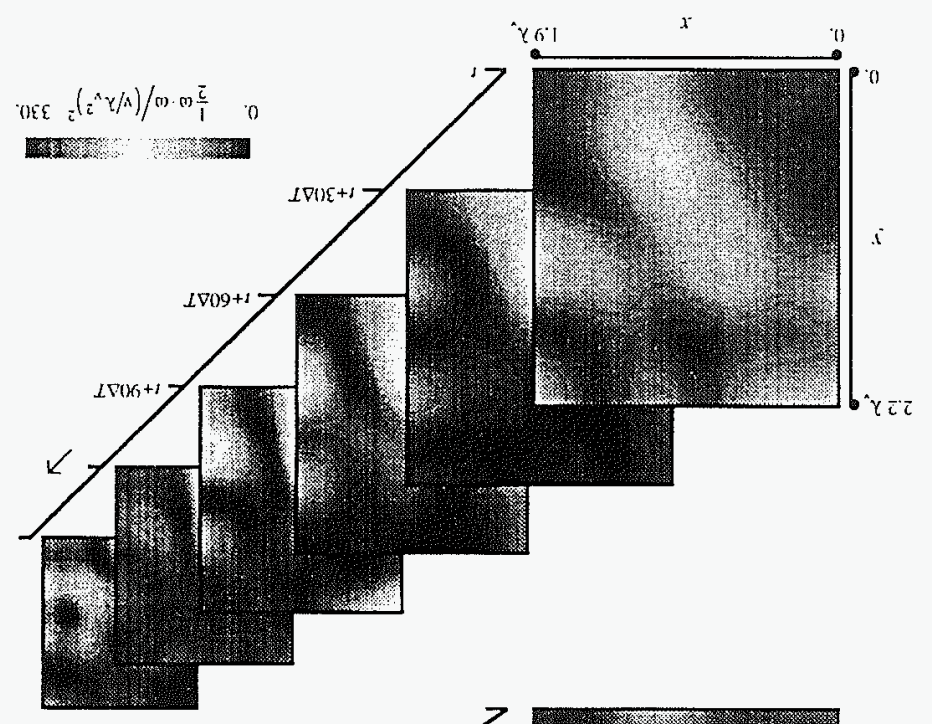

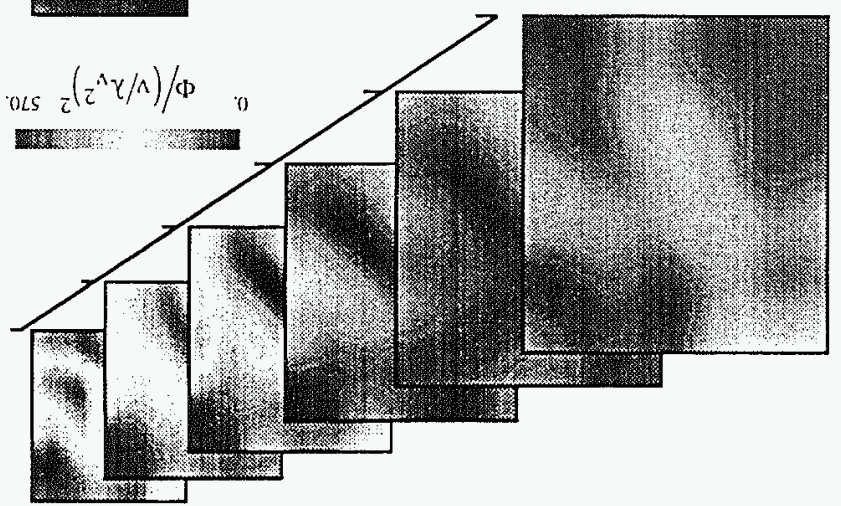


For $E$ in (5)-(8), the resulting three Euler equations are given in Su \& Dahm $(1996 a, b)$. Determination of the velocity field then involves writing these as a linear system via discrete difference operators and solving to find $\mathbf{u}(\mathbf{x}, t)$ from the given data for $\zeta(\mathrm{x}, l) ;$ e.g. with an iterative method similar to the method of conjugate gradients. It is found that the results obtained do not depend strongly on the choice of the weighting factors $\left(\alpha^{2}, \beta^{2}\right)$ over a fairly wide range of values and, owing to the integral nature of the formulation, are rather insensitive to noise in the scalar field data for values well beyond the levels in the present experimental data.

\section{Validation of the SIV Technique}

Results from a detailed DNS validation test of the SIV technique are presented by Su \& Dahm (1996a) for the extreme case of a $S_{c}=$ I scalar field in homogeneous decaying turbulence. The scalar field $\zeta(\mathrm{x}, t)$ from the DNS calculations served as input data. and the velocity field $\mathbf{u}(\mathbf{x}, t)$ obtained was cornpared with the actual DNS velocities. These showed excellent agreement of the resulting velocity field, with phase correlations exceeding $96 \%$ in the velocity field, and with probabilitydistributions for the velocity component magnitudes also showing good agreement with the corresponding DNS distributions. Moreover, in these $\mathrm{Sc} \approx \mathrm{I}$ simulations the scalar field input data contains the same amount of information as does the velocity field being sought, making the inversion extremely difficult. The agreement obtained even in this lirnitingcase suggests that, for the present experimental scalar field data with $S c$ » I, the results could be even more accurate, since then the scalar field carries far more information than does the velocity field being sought.

\section{Sample Velocity Field Measurements $\mathbf{u}(\mathbf{x}, t)$}

Figures II - I8 show sample results obtained when this SIV technique is applied to fully-resolved, four-dimensional. spatiotemporal, experimental data for $S_{c}$ » 1 scalar fields of the type in tlic previous section. In Figure I I, a scalar field plane together with the three velocity component fields in the same plane are presented. Normalization is with the local inner length scale $\lambda_{v}$ and inner velocity scale $\left(v / \lambda_{v}\right)$. These and all subsequent results have been processed with a spectrally sharp filter with cutoff wavenumber $2 \pi / \lambda_{y}$, which leaves the velocity field at scales ahove this essentially unaffected. It is apparent that the velocity field is much smoother than the scalar field, as would be expected since $\lambda_{D}=\lambda_{v} \cdot S \sim-$ "With $S c=2075$, the finest length scale in the velocity field should be 45 times larger than that in the scalar field.

Figure 12 shows a time series of the scalar field in the same plane from six spatial data volumes spaced $30 A T$ apart, where $A T$ is the temporal separation between successively acquired data volumes. The temporal spacing in Figure 12 was chosen since the advection time scale $T_{v}$ in the velocity field is 45 times longer than the advection time scale $T_{D}$ in the scalar field. Results for the three velocity component fields in each of these planes are shown in Figures $13 a, b, c$, where the evolution on this time scale can he readily seen. The probability densities in Figure I3 arc constructed from velocity fields at the roughly 300 or more time steps spanning the entire four-dimensional data space.
For each velocity vector component, results are shown from three measurements at essentially the same conditions. Note that. for each component. the three measurements give roughly similar results, though it is apparent that these statistics have not fully converged over the time spanned by any one measurement.

\section{Velocity Gradient Tensor Fields $\nabla \mathbf{u}(\mathbf{x}$, i)}

Results for all nine components of the velocity gradient tensor field are shown in Figures 14-16. Note that, unlike the velocity component statistics in Figure 13, which converge on the outer time scale $(\delta / u)$, statistics for the velocity gradient tensor components converge on the much shorter udvection time scale $T_{v}$.

The three antisymmetric components of the velocity gradient tensor field, namely the velocity vector components $\omega_{x}, \omega_{1}$. and $\omega_{z}$, are given in Figure 14. Spatial fields are shown in the same six planes as in Figure 12, as well as statistics over the entire data space from the three measurements considered. Note that statistics from two of the three cases show good agreement. The third case (R0628) is believed to have non-negligible buoyancy effects. which in inner variables simply produces a rescaling of $\lambda$. Accordingly, a single revised $\lambda_{v}$ value is used here and throughout the results presented in all following figures for this case. This brings the vorticity distributions, and the distributions for all other velocity gradient quantities. into good agreement with the two other cases.

Results for the six symmetric components of the velocity gradient tensor field. namely the three normal components $E$, $\varepsilon_{y y}$, and $\varepsilon_{z z}$ and the three shear components $\varepsilon_{x y}, \varepsilon_{z z}$, and $\varepsilon_{z x}$ of the strain rate tensor field $\varepsilon(\mathbf{x}, l)$, are shown in Figures 15 and 16. Note that statistics obtained show good agreement among the three measurements considered, and from comparisons among the various tensor components suggest only weak departures from isotropy on these scales. Of key interest for physical models of the dissipative structure of turbulence are the relative magnitudes of the eigenvalues of the strain rate tensor, and the orientation of the vorticity and scalar gradient vectors relative to the eigenvectors of the strain rate tensor field. Results of this type are experimentally accessible from SIV measurements such as these: e.g. see Su \& Dahm (19960).

\section{Turbulence Dynamics Fielcls}

The velocity gradient tensor components above are of interest in part because of their relation to various higher-order constructs associated with the dynamics of turbulent flows. Among these are such quantities as the true kinetic energy dissipation rate field $\Phi(\mathbf{x}, t) \equiv 2 \vee \mathrm{E}: \varepsilon(\mathbf{x}, t)$, the enstrophy field $1 / 2 \omega \cdot \omega(\mathbf{x}, t)$, and the enstrophy production rate field $\omega \cdot \varepsilon \cdot \omega(\mathbf{x}, t)$. Sample results for such quantities are shown in Figure 17, including spatiotemporal structure and dynamics as well as statistics.

Note that these higher-order quantities become increasingly intermittent. The dissipation and enstrophy fields, both of which are second-order in the velocity gradient tensor components, show nearly lognormal distributions. The enstrophy production rate, which is third-order, is even more highly intermittent. Moreover, note that the same rcscaled values of $\lambda_{v}$ used 
throughout these results for Case R0628 produces good agreement with the two other cases for which measurements are presented here. It should be noted that these and many other quantities obtained from these SIV measurements show good agreement with available data from numerical simulations and experimental measurements (see Su \& Dahm 1996b), providing further validation of the results obtained.

Note that, while the range of spatial scales accessible by these results is limited to about $2 \lambda_{v}$, the much longer temporal dimension of these four-dimensional spatio-temporal data provides access to inertial scales. This in turn allows such constructs as inertial range scaling exponents to be assessed from these data (e.g. Su \& Dahm 1996b) for comparisons with various theoretical predictions, large eddy and direct numerical simulations, and with other experimental measurements. Results of this type show, for example, good agreement in structure function exponents up to $14-t / h$ order with previous measurements, providing further evidence for the validity of the results obtained with this SIV technique.

\section{Pressure Gradient Fields $\nabla p(\mathbf{x}, t)$}

As a final example, Figure 18 shows experimental results for the pressure gradient vector field $\nabla p(\mathbf{x}, t)$ obtained from these velocity measurements. These are obtained by inverting the NavierStokes equation for the velocity and velocity gradient fields from the previous section as

$$
\nabla p(\mathbf{x}, t)=-\left[\frac{\partial}{\partial t}+\mathbf{u} \cdot \nabla-\frac{1}{k^{2} e} \nabla^{2}\right] \mathbf{u}(\mathbf{x}, t),
$$

where, for the present constant density case. the density has been absorbed into the pressure. This involves only spatial and temporal derivatives of the velocity field, which are fully accessible from the present measurements.

\section{WHOLE-FIELD SCALAR IMAGING VELOCIMETRY}

The four-dimensional scalar and velocity field measurements described above are fully-resolved, having spatial resolution in the scalar field finer than the local inner scale $\lambda_{D}$ and temporal resolution finer than the local advection scale $T_{\mathrm{D}}$, and in the velocity field having spatial and temporal resolution much finer than the local inner scale $\lambda_{v}$ and local advection scale $T_{v}$. However, because the Schmidt number $S c$ of the scalar is very large, and the resolution scales in the velocity and scalar fields differ by the factor $S c^{1 / 2}$. Thus the velocity field measurements obtained in the previous section are over-resolved by a factor of 45 . This makes relatively inefficient use of the spatial dynamic range available by the photodiode array, and inefficient use of the temporal dynamic range available by the total storage capacity of the measurement system. In principle, the ideal resolution for fullyresolved velocity measurements would be slightly finer than the Nyquist limit relative to $\lambda_{v}$ and $T_{v}$ (rather than $\lambda_{\mathrm{D}}$ and $T_{\mathrm{D}}$ )

It is thus natural to inquire if velocity fields can be accurately obtained from intentionally under-resolved scalar field data, in order to make use of the full dynamic range accessible to the measurements. If so, then by setting the resolution in the scalar field measurements at $\lambda_{v}$ and $T_{v}$ it would be possible to obtain fully-resolved velocity field data with a spatial dynamic range 45

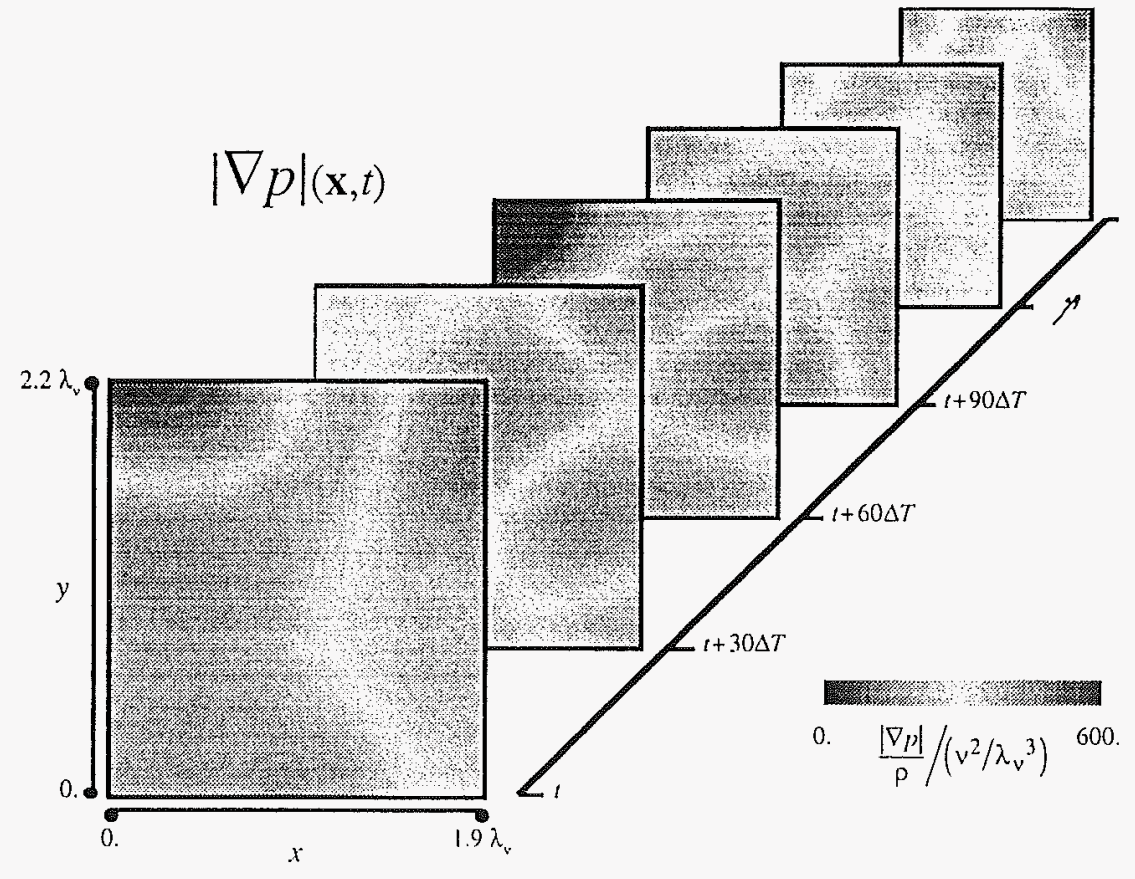

Figure IX. The pressure gradient field $\nabla p(\mathrm{x}, t)$ in the same six planes for which results were shown in the previous figures. obtained from the present scular inuging velocimetry result $\mathbf{u}(\mathbf{x}, 1)$ and the Nuvier-Stokes equation as in (10). 
times larger than in the previous section. Moreover, if the resolution in the scalar field measurements were intentionally made coarser than $\lambda_{v}$ and $T_{v}$, then true whole-field four-dimensional velocity measurements would be possible, with the spatial dynamic range of the array reaching from the local outer scale 6 to a cutoff scale determined by the spatial dynamic range of the imaging array. Equally important, since the measurements would no longer require full resolution of all spatial or temporal scales. results could be obtained at arbitrarily high outer-scale Reynolds numbers $R e_{\delta}$

To assess the feasibility of such whole-field SIV measurements, we write (I) with the aid of the continuity constraint $\nabla \cdot \mathbf{u}(\mathbf{x}, t) \equiv 0$ as

$$
\nabla \cdot \zeta \mathbf{u}=-\left[\frac{\partial}{\partial t}-\frac{1}{\operatorname{ReS} C} \nabla^{2}\right] \zeta(\mathbf{x}, t)
$$

Since any under-resolved quantity $(f\rangle_{\Delta}(\mathbf{x}, t)$ can be represented by the convolution of its resolved counterpart $f(\mathrm{x}, t)$ with a filter function $g_{\Delta}(\mathbf{x}, t)$ having spatial (temporal) scale $\mathbf{A}$ as

$$
\langle f\rangle_{\Delta}(\mathbf{x}, t) \equiv \int_{\left(\mathbf{x}^{\prime}, t^{\prime}\right)} f\left(\mathbf{x}-\mathbf{x}^{\prime}, t-t^{\prime}\right) g_{\Delta}\left(\mathbf{x}^{\prime}, t^{\prime}\right) d^{3} \mathbf{x}^{\prime} d t^{\prime},
$$

and since convolution and differentiation commute for filter functions that are symmetric in both space and time, (I I) can be written as

$$
\nabla \cdot\langle\zeta \mathbf{u}\rangle_{\Delta}=-\left[\frac{\partial}{\partial t}-\frac{1}{R e S c} \nabla^{2}\right]\langle\zeta\rangle_{\Delta}(\mathrm{x}, t)
$$

Comparing (13) with (I I) shows that straightforward application of SIV to under-resolved scalar field data produces. in place of the filtered velocity field $\langle\mathbf{u}\rangle_{\Delta}(\mathbf{x}, t)$, the scalar-weighted filtered velocity field $\langle\zeta \mathbf{u}\rangle_{\Delta} /\langle\zeta\rangle_{\Delta}$.

This scalar-weighted filtered velocity field has various physical meanings depending on the choice of filter scale A relative to the local inner length scale and advective time scale for the scalar and velocity fields, $\left(\lambda_{D}, T_{D}\right)$ and $\left(\lambda_{v}, T_{v}\right)$ respectively, and relative to the local outer scales $(\delta, u)$. Note in particular that when $\left(\lambda_{D}, T_{D}\right) \ll A \ll\left(\lambda_{v}, T_{v}\right)$ the velocity field is fully-resolved but the scalar field is under-resolved. It is then easy to show that $\langle\zeta \mathbf{u}\rangle_{\Delta} /\langle\zeta\rangle_{\Delta} \rightarrow \mathbf{u}(\mathbf{x}, t)$, so that the SIV result gives the true velocity field $\mathbf{u}(\mathbf{x}, t)$. On the other hand, when $\left(\lambda_{v}, T_{v}\right)<\mathbf{A} u(\delta, u)$ it can be shown that $\langle\zeta \mathrm{u}\rangle_{\Delta} /\langle\zeta\rangle_{\Delta} \rightarrow\langle\mathrm{u}\rangle_{\Delta}(\mathbf{x}, t)$, so that the SIV result gives the true velocity field filtered in space and time at the scale A. This is analogous to the result from a large eddy simulation (LES), but without the need for a subgrid scale model.

The suggests that physically meaningful velocity field results can be obtained via SIV at arbitrarily high values of $R e_{\delta}$. Since $\left(\lambda_{v}, T_{v}\right)$ will decreaseas the Reynolds number increases, the velocity fields are filtered at different levels, somewhat like varying the grid cutoff in an LES calculation at differing scales. When the Reynolds number is high enough that the cutoff scale is in the inertial range. such results will provide insights into the flow structure and dynamics for subgrid scale model development. Moreover. this information will be obtained in real, inhomogeneous, anisotropicturbulent shear flows, at Reynolds numbers that far exceed those accessible by DNS studies.
Perhaps most importantly. the extension of SIV to underresolved scalar field data removes the need for fully-resolved measurements of the type presented here. This would make four-dimensional spatio-temporal measurements of vector velocity fields similar to those in Figures $13-18$ possible in essentially all turbulent flows. The SIV technique would thus represent a practical, general purpose, whole-field measurement technique permitting experimental access to velocity and velocity gradient fields in essentially any flow where a conserved scalar field can be measured.

\section{CONCLUSIONS}

This paper has summarized methods for four-dimensional measurernents of conserved scalar fields and velocity fields in turbulent flows, and presented results from a number of such measurements. These allow direct experimental access to the structure and dynamics of the full scalar gradient vector field $\nabla \zeta(x, t)$ and the full velocity gradient tensor field $\nabla \mathbf{u}(\mathbf{x}, t)$ throughout a small three-dimensional spatial volume in the Flow. Moreover, the extension of these techniques currently underway to wholefield measurements, spanning from the local outer scale 6 to an inertial cutoff scale $\lambda$ set by the spatial dynamic range of the imaging array, will allow similar data to be obtained at essentially arbitrary Reynolds numbers, and in essentially arbitrary flows. Such scale-based techniques provide an alternative to particle-based imaging methods, and allow high-resolution measurements in fully-three-dimensional volumes with current detector technology.

\section{Acknowledgements}

Various aspects of the work presented here have been supported by the Air Force Office of Scientific Research under AFOSR Grant Nos. 89-054 I, 91-0402, F49620-92-J-0025 and F4962095-1-01 15. by the Gas Research Instituteunder GRI Contract Nos. 5087-260-1443 and 5093-260-2728, and with discretionary funds provided by The University of Michigan. Dr. Lester K. Su was supported during his doctoral studies at Michigan by an FXB Fellowship from the Association FXB. and Kathleen M. Tacina is supported by an NSF Fellowship at Michigan. The scalar field data were obtained at Michigan as part of the dissertation work of Dr. Kenneth B. Southerland.

\section{References}

Adrian, R.J. (1986) Multi-point optical measurements of simultaneous vectors in unsteady flow. Int. J. Heat Fluid Flow 7, 127.

Adrian, R.J. (199I) Particle imaging techniques for experimental tluid mechanics. Annu. Rev. Fluid Mecli. 2 3, 261.

Adrian, R.J. (1996) Strategies for imaging flow fields. AlAA Paper No. 96-1988. AIAA, Washington, D.C.

Balint, J.-L., Wallace, J.M. \& Vukoslavcevic, P. (1991) The velocity and vorticity vector fields of a turbulent boundary layer. Part 2. Statistical propertics. J. Fluid Meclr. 228, 53-86.

Buch, K.A. and Dahm. W.J.A. (1996a) Fine scale structure of conserved scalar mixing in turbulent flows. Part I. Sc»1. To appear in J. Fluid Mech. 
Buch, K.A. and Dahm, W.J.A. (1996b) Fine scale structure of conserved scalar mixing in turbulent tlows. Part II. $S c=1$. To be submitted to J. Fluid Mech.

Dahın, W.J.A., Southerland. K.B. \& Buch, K.A. (1991) Direct, high-resolution. four-dimensional measurements of the tine scale structure of $S C$ » I molecular mixing in turbulent flows. Phys. Fluids A 3, III5.

Dahm, W.J.A., Su, L.K. \& Southerland, K.B. (1991) Scalar imaging velocinietry studies of turbulent tlow structure and $\mathrm{dy}$ namics. Bull. Am. Phys. Soc. 36 (I0), 2674.

Dahm, W.J.A., Su, L.K. \& Southerland, K.B. (1992) A scalar imaging velocimetry technique for fully resolved fourdimensional vector velocity field measurements in turbulent tlows. Phys. Fluids A 4.2191.

Dracos, Th.A., Merkel, G.J., Rys. F., Rys, P., Maas, H.-G. \& Gruen, A.W. (1995) Velocity field measurement using laser induced fluorescence tomography. Proc. $\operatorname{Int}^{\prime} l$. Workshop on $P I V$, Fukui, Japan.

Foss, J.F., \& Wallace,J.M. (1989) The measurement of vorticity in transitional and fully-developed turbulent flows. In $A d$ vances in Fluid Mechanics (ed. M. Cad-el-Hak), Lecture Notes in Engineering 45, 263-321, Springer, Berlin.

Friehe, C.A., Van Atta. C.W. \& Gibson, C.H. (1971) Jet turbulence: dissipation rate measurements and correlations. AGARD Turbulent Shear Flows, CP-93, pp. 18-1 to 18-7.

Jiménez, J., Wray, A.A., Saffnian, P.G., and Rogallo, R.S. (1993) The structure of intense vorticity in isotropic turbulence. J. Fluid Mech. 255, 65-90.

Kasagi, N. \& Matsunaga, A. (1995) Three-dimensional particletracking velocimetry measurement of turbulence statistics and energy budget in a bnckward facing step. Int. J. Heal \& Fluid Flow $16,477-485$.

Kit, E., Tsinober, A., Balint, J.L., Wallace, J.M., \& Levich, E. (1987) An experimental study of helicity related properties of a turbulent flow past a grid. Phys. Fluids 30, 3323-3325.

Kit. E., Tsinober. A., Teitel, M., Balint, J.L., Wallace. J.M., \& Levich. E. (1988) Vorticity measurements in turbulent grid tlows. Fluid Dyn. Res. 3, 289-294.

Kovasznay, L.S.G. (1954) Turbulence measurements. In Physical Measurements in Gas Dynamics and Combustion, Vol. I0. (eds. R.W. Landenbuerg. B. Lewis, R.N. Pease \& H.S. Taylor), Princeton University Press.

Lauterborne, W. \& Vogel, A. (1984) Modern optical techniques in tluid mechanics. Annu. Rev. Fluid Mech. 12, 223.

Maas, H.-G. (1993) Determination of velocity fields in flow tomography sequences by 3 -D least squares matching. Proc. 2 nd Conf. On Optical 30 Measurement Techniques, Zurich.

Meng, H. \& Hussain, F. (1995) Instantaneous tlow field in an unstable vortex ring using holographic particle velocimetry. Phys. Fluids 7, 9.

Merkel, G.J. (1995) Tomographie in eineni turbulenten Freistrahl mit Hilfe von pH-abhängiger Lnscr Induzierter Fluoreszenz. Ph.D. Thesis No. 11 174, Eidgenössische Technische Hochschule Zürich, Switzerland.

Merkel, G.J., Rys, P., Rys, F.S. \& Dracos, Th.A. (1995) Concentration and velocity field measurements in turbulent flows by Laser Induced Fluorescence Tomography. Proc. EUROMECH Workshop on lmaging Techniques and Analysis in Fluid Dynamics, Rome; also Proc. 7 th lnt'l. Symp. on Flow Visualization, Seattle.

Miles, R.B. \& Nosenchuck, D.M. (1983) Three-dimensional quantitative flow diagnostics. In Advances in Fluid Mechanics Measurements (M. Gad-el-Hak, ed.), Lecture Notes in Engineering, Springer, Berlin.

Nishino, K., Kasagi, N. \& Hirata, M. (1989) Threedimensional particle tracking velocimetry based on automated digital image processing. J. Fluids Eng. 11 1, 384.

Pearlstein, A.J. \& Carpenter. B.N. (1995) On determination of solenoidal incompressible velocity fields from measurements of passive or reactive scalars. Phys. Fluids 7, 754.

Scherer, J. \& Bernal, L.P. (1993) Resolution characteristics of holographic particle image velocimetry. AIAA J. 31, 434.

Southerland, K.B. \& Dahm, W.J.A. (1994) Fully-resolved four-dimensional measurements of the small-scale structure of passive scalar mixing in turbulent flows. Report No. 026779 . 12, The University of Michigan. Ann Arbor.

Southerland, K.B. \& Dahm, W.J.A. (1996) Fully-resolved four-dimensional measurements of the small-scale structure of passive scalar mixing in turbulent flows. Submitted to J. Fluid Mech.

Southerland, K.B.. Dahm,W.J.A. \& Dowling, D.R. (1995) Experimental results for the high wavenumber spectral structure of scalar mixing in turbulent shear flows. Proc. 10th Symp. Turb. Shear Flows, pp. 28-1 - 27-6, Penn. State University.

Su, L.K. \& Dahni, W.J.A. (1996a) Scalar imaging velocimetry measurements of the velocity gradient tensor ficld at the dissipative scales of turbulent flows. Part I: Assessment of errors. To appear in Phys. Fluids.

Su, L.K. \& Dahm, W.J.A. (1996b) Scalar imaging velocimetry measurements of the velocity gradient tensor field at the dissipative scales of turbulent flows. Part II: Experimental results. To appear in Phys. Fluids.

Tokumaru, P.T. \& Dimotakis, P.E. (1995) Image correlation velocimetry. Expts. Fluids 19, I.

Tsinober, A., Kit, E., \& Dracos, T. (1992) Experimental investigation of the field of velocity gradients in turbulent flows. $J$ Fluid Mech. 242. 169-192.

Vukoslavcevic, P., Wallace, J.M. \& Balint, J.-L. (1991) The velocity and vorticity vector fields of a turbulent boundary layer. Part 1. Simultaneous measurement by hot-wire anemometry. J. Fluid Mech. 228, 25-51

Wallace, J.M. (1986) Methods of measuring vorticity in turbulent flows. Expts. in Fluids 4,61-71.

Wallace, J.M. \& Foss. J.F. (1995) The mcasurenient of vorticity in turbulent flows. Annut. Rev. Fluid Mech. 27, 469. 